\title{
WestVirginiaUniversity
}

THE RESEARCH REPOSITORY @ WVU

Graduate Theses, Dissertations, and Problem Reports

2017

\section{Signal and Schedule Functions when Pausing is the Operant}

Tyler D. Nighbor

Follow this and additional works at: https://researchrepository.wvu.edu/etd

\section{Recommended Citation}

Nighbor, Tyler D., "Signal and Schedule Functions when Pausing is the Operant" (2017). Graduate Theses, Dissertations, and Problem Reports. 6317.

https://researchrepository.wvu.edu/etd/6317

This Dissertation is protected by copyright and/or related rights. It has been brought to you by the The Research Repository @ WVU with permission from the rights-holder(s). You are free to use this Dissertation in any way that is permitted by the copyright and related rights legislation that applies to your use. For other uses you must obtain permission from the rights-holder(s) directly, unless additional rights are indicated by a Creative Commons license in the record and/ or on the work itself. This Dissertation has been accepted for inclusion in WVU Graduate Theses, Dissertations, and Problem Reports collection by an authorized administrator of The Research Repository @ WVU.

For more information, please contact researchrepository@mail.wvu.edu. 
Signal and Schedule Functions when Pausing is the Operant

\author{
Tyler D. Nighbor
}

Dissertation Submitted to the

Eberly College of Arts and Sciences at West Virginia University

in partial fulfillment of the requirements for the degree of

\author{
Doctor of Philosophy \\ in \\ Psychology
}

\author{
Kennon A. Lattal, Ph.D., Chair \\ Michael Perone, Ph.D. \\ Claire C. St. Peter, Ph.D. \\ Melissa D. Blank, Ph.D. \\ Gregory A. Lieving, Ph.D. \\ Department of Psychology
}

\title{
Morgantown, West Virginia \\ 2017
}

Keywords: Signaled reinforcement; differential reinforcement of other behavior; pausing 


\author{
ABSTRACT \\ Signal and Schedule Functions when Pausing is the Operant \\ Tyler D. Nighbor
}

Pausing, like other operants, is affected by the schedule of reinforcement for alternative responses and the antecedent discriminative stimuli that set the occasion for it to be reinforced. The effects of both of these variables on pausing were investigated further in three experiments in which key pecking by pigeons was reinforced concurrently according to, respectively, a variable-interval schedule, a differential-reinforcement-of-low-rate schedule, and a fixed-ratio schedule. The antecedent discriminative stimulus control of pausing was investigated by comparing unsignaled, briefly signaled, and fully signaled pausing contingencies in each experiment. Pigeons' time allocated to pausing in both full-and brief- signal conditions with each key-peck reinforcement schedule was lower than unsignaled-baseline and control conditions, demonstrating discriminative control of pausing. Although there were no systematic differences between the brief- and full-signal conditions, how pauses of the required duration were assimilated depended on the schedule of reinforcement for pecking. The current experiments replicated prior findings with a variable-interval schedule, and extended them by showing pauses were assimilated in those pauses already maintained under a differential-reinforcement-of-lowrate schedule in Experiment 2, and into the postreinforcement pause under a fixed-ratio schedule in Experiment 3. Together, these results demonstrate the antecedent and consequent control of pausing under several schedules of reinforcement for pecking, under which characteristically different peck-pause interactions emerge. 


\section{Acknowledgements}

I would like to thank Andy Lattal for his guidance throughout my time at West Virginia University. I would also like to thank Mike Perone, Claire St. Peter, Melissa Blank, and Greg Lieving for their helpful comments and discussion at all stages of this project. Finally, I would like to thank the graduate and undergraduate students that have been crucial to the success of this and other projects. 


\section{Table of Contents}

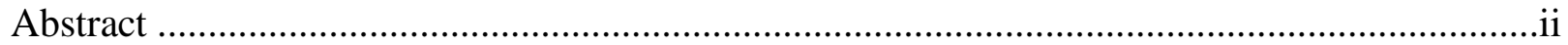

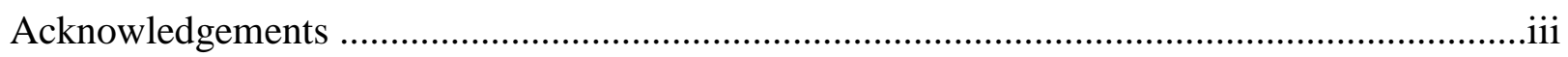

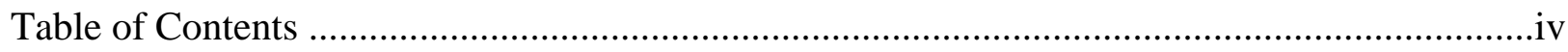

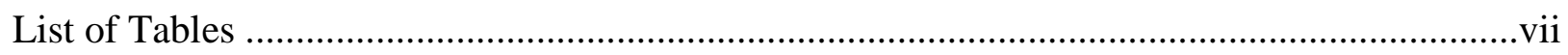

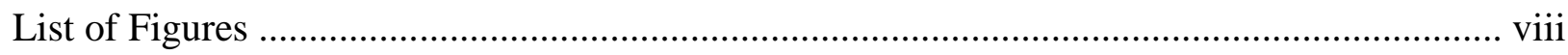

Introduction

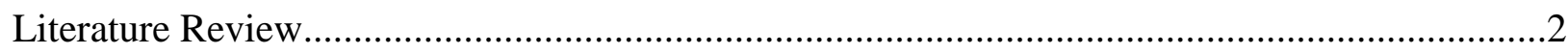

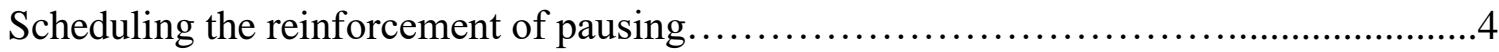

Pausing in concurrent schedules...........................................6

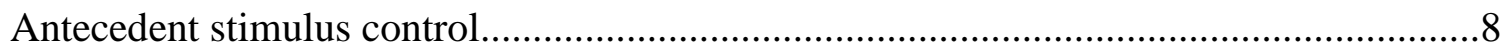

Signaled delay of reinforcement............................................ 10

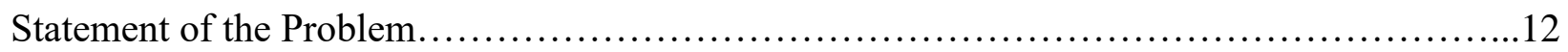

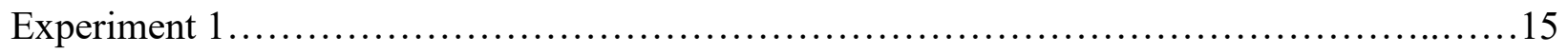

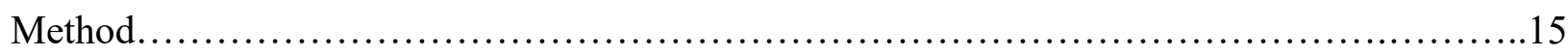

Subjects................................................................. 15

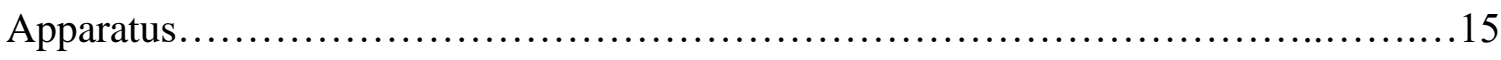

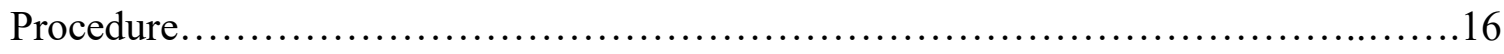

Unsignaled baseline.................................................16

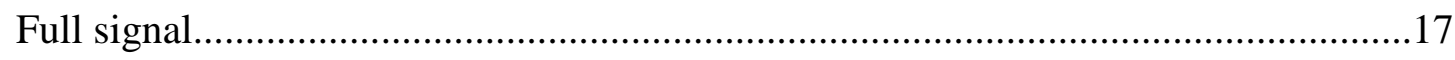

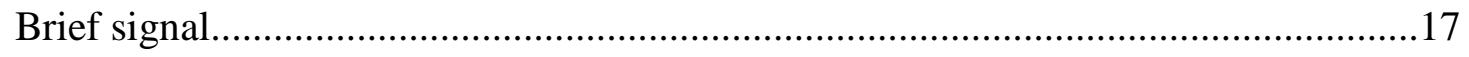

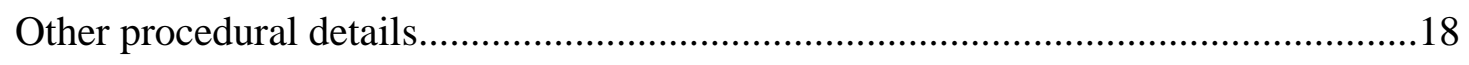

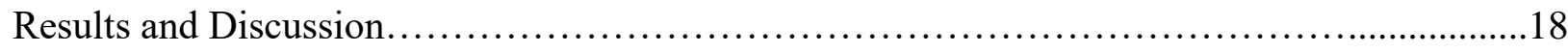




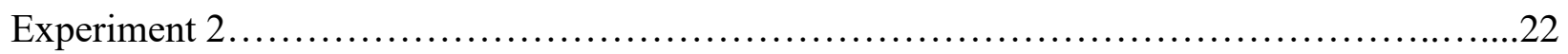

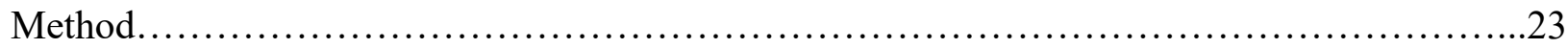

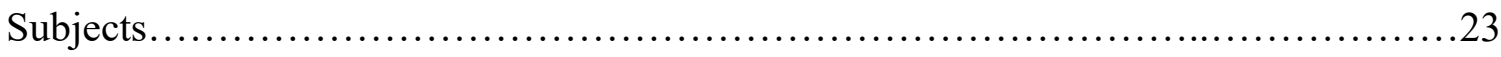

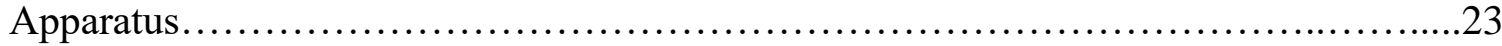

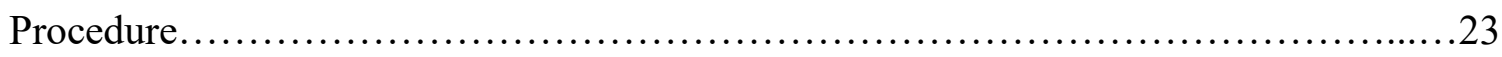

Full Signal and Brief Signal............................................24

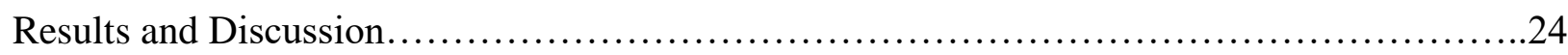

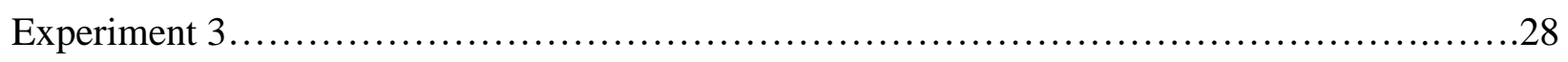

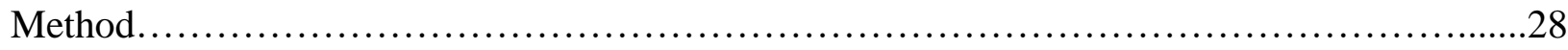

Subjects........................................................... 28

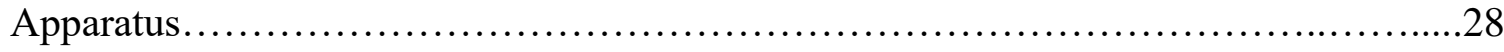

Procedure..............................................................29

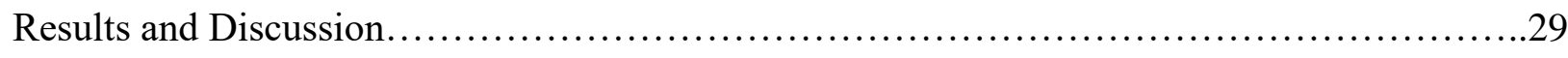

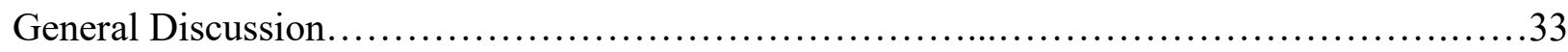

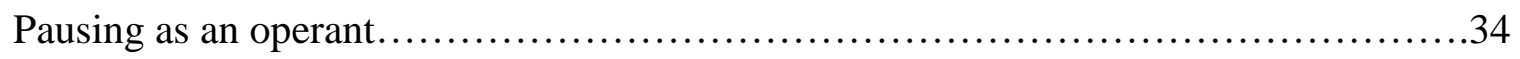

Pausing in the context of the reinforcement of a discrete response $\ldots \ldots \ldots \ldots \ldots \ldots \ldots \ldots . \ldots . \ldots . \ldots 5$

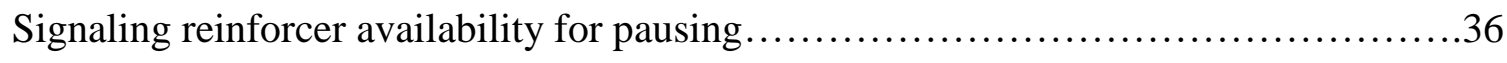

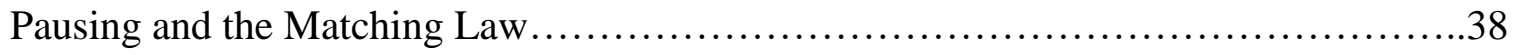

Potential Applications......................................................40

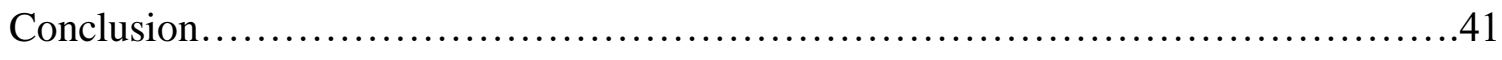

References.................................................................... 42 


\section{List of Tables}

Table 1. Experiment 1 Stimuli. Standard color refers to the key color corresponding with the respective multiple schedule component and Signal color refers to the key color during the during either the 1) DRO portion of the chain VT DRO schedule or 2) stimulus change in the

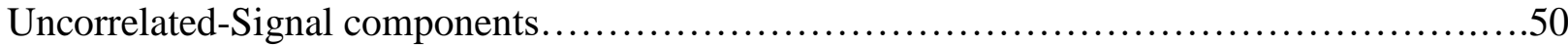

Table 2. Experiment 1 order of conditions and number of sessions for individual pigeons. $\mathrm{U}=$ Unsignaled-Baseline condition, $\mathrm{F}=$ Full-Signal condition, $\mathrm{B}=$ Brief-Signal condition. ....

Table 3. Experiment 2 Stimuli. Standard color refers to the key color corresponding with the respective multiple schedule component and Signal color refers to the key color during the during either the 1) DRO portion of the chain VT DRO schedule or 2) stimulus change in the

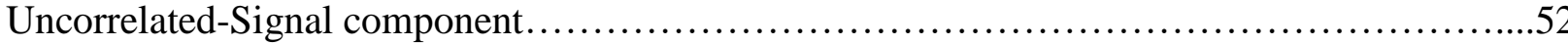

Table 4. Experiment 2 order of conditions and number of sessions for individual pigeons. $\mathrm{U}=$ Unsignaled-Baseline condition, F = Full-Signal condition, B = Brief-Signal condition...........53

Table 5. Experiment 3 Stimuli. Standard color refers to the key color corresponding with the respective multiple schedule component and Signal color refers to the key color during the during either the 1) DRO portion of the chain VT DRO schedule or 2) stimulus change in the

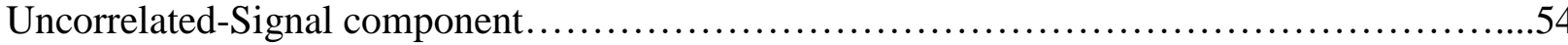

Table 6. Experiment 3 Order of conditions and number of sessions for individual pigeons. $\mathrm{U}=$ Unsignaled-Baseline condition, F = Full-Signal condition 


\section{List of Figures}

Figure 1. Percentage of session time in Experiment1 allocated to pausing in the UnsignaledBaseline (U), Brief-Signal (B), and Full-Signal conditions (F) for the last ten sessions, separated by Correlated-Signal (Co) and Uncorrelated-Signal (Un) components, for individual pigeons.56

Figure 2. Response rates from Experiment 1 in the Unsignaled-Baseline (U), Brief-Signal (B), and Full-Signal conditions (F) for the last ten sessions, separated by Correlated-Signal (Co) and Uncorrelated-Signal (Un) components, for individual pigeons $\ldots \ldots \ldots \ldots \ldots \ldots \ldots \ldots \ldots \ldots \ldots . .57$

Figure 3. Mean reinforcement rates from Experiment 1 for the pecking (VI) and pausing (DRO) contingencies in the Unsignaled-Baseline (U), Brief-Signal (B), and Full-Signal conditions (F), separated by Correlated-Signal (Co) and Uncorrelated-Signal (Un) components. Error bars are

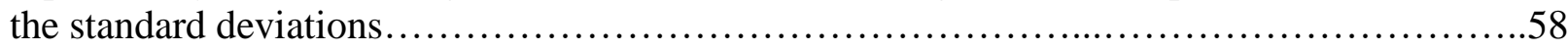

Figure 4. Log-survivor plots of IRTs from Experiment 1, separated by Correlated-Signal (Co) and Uncorrelated-Signal (Un) components during the Unsignaled-Baseline conditions, in the left panel, and from Full-Signal (F) and Brief-Signal (B) conditions, in the right panel.

Figure 5. Percent time allocated to pausing from Experiment 2 in the Unsignaled-Baseline (U), Brief-Signal (B), and Full-Signal (F) conditions for the last ten sessions, separated by CorrelatedSignal (Co) and Uncorrelated-Signal (Un) components, for individual pigeons (y -axis starts at $50 \%)$.

Figure 6. Response rates from Experiment 2 in the Unsignaled-Baseline (U), Brief-Signal (B), and Full-Signal conditions (F) for the last ten sessions, separated by Correlated-Signal (Co) and Uncorrelated-Signal (Un) components, for individual pigeons..........................60

Figure 7. Mean reinforcement rates from Experiment 2 for the pecking (DRL) and pausing (DRO) contingencies in the Unsignaled-Baseline (U), Brief-Signal (B), and Full-Signal (F) conditions, separated by Correlated-Signal (Co) and Uncorrelated-Signal (Un) components. Error bars are the standard deviations.

Figure 8. Log-survivor plots of IRTs from Experiment 2, separated by Correlated-Signal (Co) and Uncorrelated-Signal (Un) components during the Unsignaled-Baseline conditions, in the left panel, and from Full-Signal (F) and Brief-Signal (B) conditions, in the right panel...........62

Figure 9. Percent session time allocated to pausing from Experiment 3 in the UnsignaledBaseline (U) and Full-Signal (F) conditions for the last ten sessions, separated by CorrelatedSignal (Co) and Uncorrelated-Signal (Un) components, for individual pigeons....

Figure 10. Response rates from Experiment 3 in the Unsignaled-Baseline (U) and Full-Signal (F) conditions for the last ten sessions, separated by Correlated-Signal (Co) and Uncorrelated-Signal

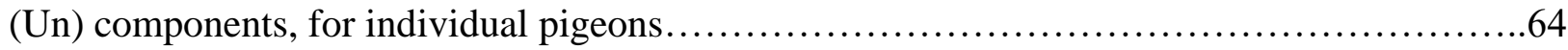

Figure 11. Run rates from Experiment 3 in the Unsignaled-Baseline (U) and Full-Signal (F) conditions, separated by Correlated-Signal (Co) and Uncorrelated-Signal (Un) components, for individual pigeons 
Figure 12. Mean postreinforcement pauses from Experiment 3 in the Unsignaled-Baseline (U) and Full-Signal (F) conditions, separated by Correlated-Signal (Co) and Uncorrelated-Signal (Un) components, for individual pigeons. Error bars are the standard deviations

Figure 13. Mean reinforcement rates from Experiment 3 for the pecking (FR) and pausing (DRO) contingencies in the Unsignaled-Baseline (U) and Full-Signal (F) conditions, separated by Correlated-Signal (Co) and Uncorrelated-Signal (Un) components. Error bars are the standard

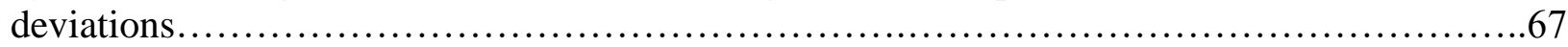

Figure 14. Log-survivor plots of IRTs from Experiment 3, separated by Correlated-Signal (Co) and Uncorrelated-Signal (Un) components during the Unsignaled-Baseline conditions, in the left panel, and from the Full-Signal (F) condition, in the right panel...........................68 


\section{Introduction}

Noting that no two responses are the same and that a reproducible unit is a prerequisite to developing a science of behavior, Skinner (1935) conceptualized the operant class, which he defined in terms of individual responses that are evoked under similar stimulus conditions and have common effects on the environment. Operants have been defined both molarly, as an aggregate of responding across a period of time (i.e., response rate, or even a response pattern), and molecularly, as, for example, the time between two successive responses (i.e., interresponse times). An operant also can be a period of nonengagement with respect to a target response, as in the case of differential reinforcement of other behavior (DRO; Reynolds, 1961; see also Craig, Lattal, \& Hall, 2014; Lattal \& Boyer, 1980). By definition, DRO schedules reinforce responses other than the target response; however, this "other" behavior is a construct describing inferred behavior that, as suggested by the phrase "DRO," is increased. The parameters of "other" behavior are uncertain; however, an alternative description may be that DRO schedules most explicitly reinforce periods of nonengagement, or "pausing," in a predefined target response. If put in the context of another operant defined by commission (e.g., pecking), the relation between pausing and pecking is orderly, conforming to the generalized matching law (Baum, 1974; 1979; Craig et al., 2014; Lattal \& Boyer, 1980). In the following three experiments, such "other" behavior was investigated.

Like any other operant, pausing is affected by both the schedules of reinforcement of concurrently available alternative responses (Craig et al., 2014; Lattal \& Boyer, 1980) and the antecedent discriminative stimuli, or signals, that set the occasion for responses to be reinforced (Craig et al., 2014; Exp. 2). In the present experiments, both of these variables were investigated further. The literature review that follows first addresses conceptual issues surrounding "other" 
behavior. Next, research as it relates to the understanding of "other" behavior as an operant is presented. Finally, the role of signals as discriminative stimuli are reviewed.

\section{Literature Review}

Operants occur in the context of other concurrently available alternative operants (Herrnstein, 1970). A concurrent schedule often is arranged in the laboratory in which two or more response alternatives are simultaneously and independently reinforced, either by responding on different operanda (Catania, 1963), or by responding on a single operandum under the control of different discriminative stimuli (Findley, 1958). Using pigeons and concurrent variable-interval (VI) VI schedules, for example, Herrnstein (1961; 1970; see also Baum 1974, 1979) showed that the proportion of responses on one alternative will equal, or match, the proportion of reinforcers delivered by that alternative. Herrnstein's original equation describing this relation later was transformed into the generalized matching law, which describes sensitivity of behavior to reinforcement-rate differences and bias for one operant over the other (Baum, 1974;1979). Baum (1979) reviewed 103 sets of data that conformed closely to the generalized matching equation.

Data described by the matching law most often are generated by the concurrent reinforcement of topographically similar response alternatives (e.g., pecking two different keys); however, in several experiments the matching law has been confirmed when the operants are topographically different. For example, McSweeney (1978) reinforced key pecking and treadle pressing concurrently under VI schedules and found that the matching law described both responses, but responding was biased toward key pecking. Similarly, Sumpter, Foster, and Temple (1998) arranged concurrent VI VI schedules in which key pecking and door pushing by hens were reinforced. Again, the matching law described the time allocated to both responses 
with a bias for key pecking. The matching law also describes cases where the operant was not a discrete response (e.g., pecking). Baum and Rachlin (1969) placed food hoppers on either end of a long-operant chamber and reinforced standing on either side concurrently on independent variable-time (VT) schedules. Time standing on either side of a chamber conformed to the matching law.

Although what has been discussed thus far is programmed contingencies, reinforcers other than those programmed by the experimenter also affect responding. Herrnstein (1970) included the variable $r_{o}$ (sometimes alternatively labeled $r_{e}$ ), or an unknown aggregate of reinforcement for "other" alternative responses, to suggest how reinforcement from "other" sources interacts with operant sources of reinforcement in controlling responses and response allocation between alternatives. Herrnstein's equation predicts that responding associated with the programmed contingency will be inversely related to responding related to alternative sources of reinforcement for “other" behavior. One issue, however, with Herrnstein's $r_{o}$ is that it aggregates all the other reinforcement sources into a single variable. Thus, it is not possible to parse apart the relative contributions of spilled food on the ground and preening to the aggregate. It also is difficult to compare reinforcers associated with activities like "preening" to 3-s access to food programmed for key pecking. Another issue with $r_{o}$ is that it is the result of an almost unlimited range of alternative responses (e.g., grooming, spinning) that may be quite variable. Perhaps another way to study "other behavior" is to place it in the context of another measured response and define it as periods of nonengagement, or "pausing," in the measured response. Whereas Herrnstein discussed $r_{o}$ as a free parameter to help account for deviations from matching, pausing may be arranged as a different operant akin to concurrently reinforcing 
operants that are topographically different from one another (McSweeney, 1978; Sumpter et al., 1998).

\section{Scheduling the reinforcement of pausing}

Schedules of reinforcement like fixed ratio (FR) and fixed interval (FI) generate pausing by the nature of the contingencies they impose. The patterns of responding on FR and FI schedules are described as, respectively, "break and run" and "scalloped," reflecting the fact that following food delivery, a period of nonresponding, or postreinforcement pause (break) typically occurs, followed by either a relatively rapid or gradually increasing transition to a high rate of responding (Ferster \& Skinner, 1957; Lattal, 1991). A number of variables affect the length of the postreinforcement pause, such as the size of the ratio or length of interval (Ferster \& Skinner, 1957; Felton \& Lyon, 1966; Innis, Mitchell, \& Staddon, 1993; Powell, 1968; Schneider, 1969) and magnitude of the reinforcer (e.g., Grace \& Nevin, 2000; Perone \& Courtney, 1992; Powell, 1969; Staddon, 1970).

In the above schedules, pausing emerges from other contingencies associated with the schedule, but pausing also may be reinforced explicitly. The DRO schedule is an example of the latter. In DRO schedules, reinforcement depends explicitly, and solely, on the absence of a target response. Several observers (e.g., Uhl \& Garcia, 1969; Zeiler, 1971, 1976; 1977) have taken issue with the label "DRO," as it carries surplus meaning. The label implies not that the absence of responding is reinforced, but instead that "other" unobserved and unmeasured responding is reinforced. This is problematic in that the definition of this schedule includes a "theoretical account of its method of action instead of an objective description of the dependency" (Zeiler, 1971, p. 22). Additionally, the absence of the target response may have functional properties that meet the requirements of an operant (Zeiler, 1972). Because of these issues, several alternative 
labels to DRO have been proposed, including "differential reinforcement of pausing" (DRP; Kelleher, 1961; Zeiler, 1976), “omission training” (Uhl \& Garcia, 1969), and “not-R > t," where $t$ is a measure of time (Schoenfeld, Farmer, \& Vickery, 1970). These alternative labels, like most (but not all) other schedule labels, refer to the experimental operations involving the responsereinforcer dependencies (i.e., a programmed reinforcer is not delivered following the target response) rather than the assumption that "other" behavior is reinforced (Kelleher, 1961; Lane, 1961; Uhl \& Garcia, 1969). Conversely, it could be argued that conceptualizing behavior as a negative event, that is, the nonoccurrence of a response, is vague and imprecise, placing it at odds with a science of behavior that emphasizes precision. Behavior in the negative case, however, is precise when nonengagement is measured as time allocation with respect to a discrete, precisely defined target response (e.g., key pecking). Additionally, the DRO contingency most explicitly depends on the absence of the target response. Although theoretically the organism may be actively doing "something else," that something else could be, in the best Seinfeldian tradition, "doing nothing" and still meet the reinforcement requirement.

Counter to the above issues is the observation that the DRO vocabulary is well established (Catania, 2007). A slight twist in the definition of the abbreviation, however, allows one to keep one's label and change it, too. Lane (1961. p. 175) described DRO as "differential reinforcement of zero [target response] rates" or of "zero [target] responses." Because differential reinforcement of other behavior involves both the hypothetical construct of other behavior and an unsubstantiated mechanism by which the construct is maintained, this slight twist on the label "DRO" allows its use to be consistent with most other procedural descriptions of reinforcement schedules. Thus, DRO can be taken to describe, not other behavior, but pauses (zero rates) in the target response. 


\section{Pausing in concurrent schedules}

Pausing in the steady state is not very interesting to study. After all, zero rates of the target response are always zero rates of the target response. So, again, in Seinfeldian mode, the study of DRO in the steady state is the study of nothing. When, however, the occurrence and the nonoccurrence of the same response are eligible for reinforcement concurrently, the target response may be modulated rather than eliminated as a function of the parameters of either schedule (Rachlin \& Baum, 1972; Zeiler, 1976; Zeiler \& Fite, 1975). The effects reported to date resemble those found when a punisher and a positive reinforcer are scheduled concurrently for the same response. They suggest that positive reinforcers made dependent on zero target responses, or target-response omission, have effects like those of other response-dependent punishers.

When a DRO schedule is arranged as the sole contingency in effect, reinforcement rate and pause requirements are negatively correlated with one another. In other words, increasing the pause requirement also decreases reinforcement rate, confounding the two and making it difficult to draw conclusions about the relative contribution of either of these variables to the target response rate reductions. To attenuate this, Nevin (1968) used chain VI (20-s to 3-min) DRO 10$\mathrm{s}$ schedules in which reinforcers first became available following a varying period of time, signaled by a change in the keylight color, after which reinforcers were delivered following a 10$\mathrm{s}$ absence of the target response. Response rates in the VI component varied inversely with reinforcement rate. Using Nevin's procedure, Rachlin and Baum (1972) reinforced key pecks, but concurrently delivered reinforcers either independently of responding according to a VT schedule, or, similar to Nevin but without a signal (tandem schedule) indicating reinforcement availability, following the first 2-s pause after a variable-time period lapsed. Key-peck response 
rates maintained by the VI schedule varied inversely with the frequency of VT or DRO reinforcement, and the function relating response rate and reinforcement rate was described by the matching law. Rachlin and Baum thus provided the first analysis of how the alternative reinforcement of pausing interacted with the concurrent reinforcement of a target response.

Using a method different from that used by Nevin (1968) and Rachlin and Baum (1972), Zeiler (1976) studied fixed-time (FT) 10-, 20-, 30-, or 100-s or DRO 10-, 20-, 30-, or 100-s schedules arranged concurrently with FI 3-min schedules. Both concurrent arrangements reduced the rate of key-pecking proportionally to the frequency of the alternative source of reinforcement (i.e., FT or DRO). In conditions with relatively frequent alternative reinforcement, pausing functioned as other concurrently maintained responses, similar to the findings of Nevin (1968) and Rachlin and Baum (1972). Subsequently, Zeiler (1977) maintained key pecking on VI or variable-ratio (VR) schedules while concurrently reinforcing periods of nonresponding according to either DRO or FT schedules, wherein a predetermined number of consecutive pauses of a fixed duration were reinforced. Again, there was a negative relation between the pause requirement and key-peck response rates. A limitation of Zeiler's (1976; 1977) experiments, however, is that each pause requirement created a different operant, if the operant is defined as a pause of the required duration. As a result, the rate of reinforcement for pausing varied with each change in the required pause, making conclusions about relative contributions of reinforcement rate and pause duration difficult.

Lattal and Boyer (1980, Exp. 2) reinforced pausing using a procedure similar to that described by Rachlin and Baum (1972). A tandem VT $x$-s DRO 5-s schedule was programmed with a concurrently operating FI 5-min schedule reinforcing key pecking. Thus, the first 5-s pause in key-pecking was reinforced after a variable time period lapsed. Pausing was defined as 
any period of nonengagment in key-pecking $\geq 5 \mathrm{~s}$. In contrast with Zeiler $(1976 ; 1977)$ and similar to Nevin (1968) and Rachlin and Baum (1972), the pause requirement was held constant through manipulations of the reinforcer frequency for pausing. The amount of session time allocated to pausing was proportional to the relative rate of reinforcement for pausing, conforming to the predictions of the generalized matching law. There was a bias for pausing, however, perhaps related to the post-reinforcement pausing induced by FI schedules. To investigate pausing as an operant in the absence of such schedule-induced pausing, Craig, Lattal, and Hall (2014, Exp. 1) arranged a tandem VT $x$-s DRO 5-s schedule as in Lattal \& Boyer (1980, Exp. 2) concurrently with a VI instead of an FI schedule to maintain key pecking. Time allocated to pausing (as defined by Lattal \& Boyer, 1980) was proportional to the reinforcement rates associated with pausing, therefore again conforming to the matching law. With this variable availability of reinforcement for pausing, there was no systematic bias for pecking or pausing.

\section{Antecedent stimulus control}

Operant responding is controlled not only by its consequences, but also by motivating operations and antecedent discriminative stimuli that set the occasion for responses to be reinforced. Thus, there are ways in which responding can be controlled more in accord with the programmed contingency. For example, in VI schedules, a variable amount of time elapses and then only a single response is required for reinforcement. In the absence of discriminative stimuli, pecking at a steady rate maximizes reinforcement rate. Marcucella (1976) arranged a multiple VI VI schedule. In one component, the key color changed when the scheduled interreinforcer interval lapsed, providing a discriminative stimulus for key pecking (technically converting that component to a multiple VT FR1 schedule). Key pecking under the signaled schedule, unsurprisingly, was reduced to near-zero rates, as opposed to the increase in key- 
pecking rates that occurred in the other multiple schedule component where the availability of reinforcement was unsignaled. Subsequently, using a concurrent VI VI schedule, Marcucella and Margolius (1978) found that nearly all of the pigeons' time was allocated to the component where reinforcement was unsignaled as compared to the signaled component, systematically replicating Marcucella (1976).

Signaling reinforcer availability also may bring pigeons' responding more in accord with the reinforcement schedule requirements in those schedules where responding before the reinforcer becomes available interferes with optimizing reinforcement rate. Specifically, in differential-reinforcement-of-low-rate (DRL), or IRT $\geq t$, schedules, a response is reinforced only if that response occurs after $t$ (see Kramer \& Rilling, 1970, for a review). Despite the programmed contingency, responding tends to occur frequently prior to $t$ (e.g., Ferster \& Skinner, 1957; Richards, Sabol, \& Seiden, 1993; Staddon, 1965). Responding under DRL schedules also can come under discriminative stimulus control. Catania (1970) and Shimp (1968) reported that in DRL schedules with $t>30 \mathrm{~s}$, fewer responses occurred prior to $t$ when a stimulus change denoted that $t$ had elapsed. Zeiler and Blakely (1983) found that changing the keylight color when $t$ elapsed on a DRL 10-s or 20-s schedule resulted in almost all of the responses occurring after $t$, thus bringing the organism's behavior in closer correspondence to that specified by the programmed contingency.

Stimuli signaling reinforcer availability for pausing can also bring pausing under discriminative control. Weisman (1970) stabilized responding on a multiple VI 1-min VI 1-min schedule before changing the schedule to a multiple VI 1-min DRO (range 4- to 32-s) schedule in which the VI was correlated with a green keylight and the DRO with a horizontal line superimposed on the green keylight. Pausing was controlled by the horizontal line discriminative 
stimulus, in that response rates decreased in the presence of this stimulus (see also Nevin, 1968). Pausing similarly comes under the control of discriminative stimuli in concurrent schedules. Craig et al. (2014, Exp. 2) arranged reinforcement for both pecking and pausing in a twocomponent multiple schedule, each component consisting of a concurrent (VI 90-s) (tandem VT 85-s DRO 5-s) schedule (as in their Experiment 1, described above). In one component of the multiple schedule, labeled the DRO-signal component, a key-color change occurred following the lapse of the VT interval, signaling the onset of the pause contingency. The key reverted to the nonsignal color only after a 5-s pause and reinforcement occurred. In the other component, labeled the arbitrary-signal component, a key-color change of $5 \mathrm{~s}$ was controlled by a VT 85-s schedule such that the key-color change occurred independently of both pausing and pecking. Signaling the availability of reinforcement of the pause contingency decreased the amount of time allocated to pausing in the signaled component as compared to the arbitrary-signaled component, similar to the findings of Marcucella (1976) and Marcucella and Margolius (1978). In each of the experiments described in this section, then, operant pausing was controlled not only by consequent events, but also by antecedent stimuli indicating reinforcement availability.

\section{Signaled delay of reinforcement}

Skinner (1938) included a DRO contingency as a resetting delay procedure when, in developing a procedure in which a single response started a delay period controlled by timer that ended in reinforcement, he added another contingency such that "if a second response is made during the delay interval, the timing begins again, so that a full interval must again elapse before reinforcement occurs" (p. 139). Under this schedule, response rates were reduced, relative to baseline. Response rates on VI schedules in which the reinforcer occurs following an unsignaled delay between that reinforcer and the response that produced it are lower than such rates when 
the reinforcer immediately follows the response (Catania \& Keller, 1981; Sizemore \& Lattal, 1977; Williams, 1976). Associating the entire delay period with a distinct stimulus (labeled a fully signaled delay) still often reduces response rates relative to those maintained by immediate reinforcement, but the rates are higher than those maintained by otherwise equivalent unsignaled delays. Richards (1981) arranged a VI 60-s schedule (or DRL 20-s schedule for some pigeons) in baseline, and subsequently added delays ranging from $.5 \mathrm{~s}$ to $10 \mathrm{~s}$. Unsignaled delays of 5 and 10 s substantially decreased response rates, whereas signaled delays of the same values produced only moderate decreases in response rates, relative to baseline (see also Lattal, 1984).

Given the above findings regarding the absence and presence of signals, Schaal and Branch (Exp. 2) investigated whether variations of the signal duration differentially affected response rates. A multiple VI 60-s VI 60-s schedule served as the baseline. Following the addition of 3-s unsignaled delays in both components, response rates decreased below those observed in the baseline condition. Subsequently, 3-s delays in one component were signaled by a brief signal (.5-s change in key color), and in the other component, a full signal (a different key color) remained changed for the entire delay. Response rates increased to near-baseline levels in both components. This effect persisted until the delay was increased to $27 \mathrm{~s}$, at which point response rates decreased in the briefly signaled delay component but remained at near-baseline levels in the fully signaled delay component.

Schaal and Branch's (1988) findings suggest an interaction between the duration of the signal and the duration of the delay. In their procedure, the signal indicated the completion of a response requirement and was associated with reinforcer onset. Such signals also may set the occasion for responding to be reinforced, and therefore, a related question may be asked: what is the relation between signal duration and the antecedent discriminative control of pausing? When 
Craig et al. (2014, Exp 2) signaled the availability of reinforcement for pausing, pausing was reduced relative to that occurring in a component in which an equivalent signal occurred, but was uncorrelated with reinforcement availability. The Craig et al. experiment is limited in its application to signaling operant reinforcement availability because only full signals were used. Schaal and Branch (1988) showed that the signal duration had differential effects on response rates. Signal duration in the antecedent discriminative control of operant pausing, however, has not been parametrically investigated.

\section{Statement of the Problem}

Herrnstein (1970) proposed that responding associated with programmed contingencies is inversely related to alternative sources of reinforcement for “other” behavior. Herrnstein's inclusion of "other" behavior was an important early conceptualization of the role of "other" behavior in competing with programmed operant contingencies. "Other" behavior, however, was used as a free parameter to account for deviations from matching, and therefore, Herrnstein's was a conceptual rather than an experimental analysis of such behavior. Another way to study "other" behavior is to arrange for the direct reinforcement of "other" behavior by placing it in the context of a measured response and defining it as periods of nonengagement, or "pausing," in the measured response, as in the case of DRO (Reynolds, 1961). Pausing may be reinforced concurrently with a targeted discrete response (Zeiler, 1976; Zeiler, 1977), akin to the reinforcement of topographically dissimilar operants (McSweeney, 1978; Sumpter et al., 1998). Apropos to the latter, Lattal and Boyer (1980, Exp. 2) reinforced pausing with a concurrently operating FI schedule, and found the amount of session time spent pausing was proportional to the relative rate of reinforcement for pausing. There was a bias for pausing, however, perhaps related to the postreinforcement pausing induced by FI schedules. To attenuate the bias for 
pausing, Craig, et al. (2014, Exp. 1) reinforced pausing concurrently with pecking under a VI instead of an FI schedule. In this case, time allocated to pausing was proportional to the reinforcement rates associated with pausing with no systematic bias for pecking or pausing. Interval schedules (i.e., VI and FI) allow for the assimilation of pausing into the performance maintained by them when the pause is added because, depending on the size of the interreinforcer interval, such added pauses do not change reinforcement rates of the pecking contingency (Lattal \& Boyer, 1980; Craig et al., 2014). The same may not be true, however, of other schedules. One general question is how reinforcing pausing affects behavior maintained by schedules other than those imposing interval contingencies, and specifically, if reinforcing pausing disrupts the reinforcement under other contingencies. Under DRL schedules, pausing occurs, but unlike FI or VI schedules, the pause requirement in DRL schedules is specified by the schedule. A DRL schedule differs from a DRO schedule in that a pause between responses $\geq$ $t$ is required, but following the pause requirement, a response must occur before reinforcement. Thus, if too much time is allocated to pausing, or conversely, pecking, reinforcement rate under either the DRL or DRO schedule may be reduced.

In addition, like FI schedules, FR schedules involve postreinforcement.pauses, yet pausing is perhaps more compatible with FI schedules than FR schedules. Although there are places in the schedule where pauses occur naturally (i.e., the postreinforcement pause), inducing additional pausing during ratio runs in FR schedules may disrupt reinforcement rate. Thus, it is possible characteristics of the schedule of reinforcement for pecking may alter aspects of pausing as an operant.

Operant responding is controlled not only by its consequences, but also by antecedent discriminative stimuli that set the occasion for responses to be reinforced. Pausing has been 
shown to be a discriminated operant in the context of multiple schedules of reinforcement (Reynolds, 1961; Weisman, 1970). Discriminated operants also have been established through signaling reinforcement availability, which brings the operant response at least partially under the control of the signal (e.g., Marcucella, 1976; Marcucella \& Margolius, 1978). Craig et al. (2014; Exp. 2) demonstrated that pausing can be a discriminated operant through signaling reinforcer availability of pausing in the context of reinforcement for pecking under VI schedules. Aside from signals setting the occasion for responding to be reinforced, there is evidence that the proportion of a delay interval that is signaled affects the maintenance of responding when reinforcement is delayed from the response that produces it. Responding may produce a lasting change in environmental circumstances that is correlated with upcoming reinforcement, and in some cases, these changes in environmental conditions may be only momentary. Signals less than the full duration of the delay, under certain conditions, produce lower rates of responding relative to conditions in which the entire delay is signaled (Schaal \& Branch, 1988). In line with Schaal and Branch, lasting or momentary changes in environmental circumstances also may set the occasion for responding to be reinforced. It was unknown, however, if varying the signal duration would differentially affect the antecedent discriminative stimulus control, hereafter referred to as discriminative control, of pausing under conditions in which pausing was reinforced concurrently with key pecking.

In the following series of experiments, pigeons responded under a two-component multiple schedule in which signals of different durations (i.e., $.5 \mathrm{~s}$ or until reinforcer delivery) either signaled pause-dependent reinforcer availability, or were uncorrelated with such reinforcer availability. With pausing defined as a period $\geq 5$ s of nonengagment in key pecking (Craig et al., 2014; Lattal \& Boyer, 1980), the experiments were designed to compare the effects of 
unsignaled, briefly signaled, and fully signaled pausing contingencies under two different reinforcement schedules (VI and DRL in Experiment 1 and 2, respectively) and the effects of unsignaled and fully signaled pausing contingencies under an FR schedule (Experiment 3).

\section{Experiment 1}

Using a procedure similar to Craig et al. (2014), Experiment 1 was designed to compare pausing in the absence of a signal indicating reinforcer availability for pausing to that when availability was signaled either briefly or for the full duration of the required pause.

\section{Method}

\section{Subjects}

Four male White Carneau pigeons with previous experimental histories were used. Each was housed in separate cages in a vivarium under a 12:12-hr light/dark cycle. The pigeons had continuous access to water and health grit in their home cages. Each was maintained at $80 \%$ of its ad libitum body weight (+/- $15 \mathrm{~g})$ by means of postsession feedings as required.

\section{Apparatus}

The work area of the operant chamber was $32 \mathrm{~cm} \mathrm{H} \mathrm{x} 30 \mathrm{~cm} \mathrm{~W} \mathrm{x} 31 \mathrm{~cm} \mathrm{~L}$. All walls and the door were made of Plexiglas ${ }^{\circledR}$, except for the aluminum work panel. One 3-cm diameter Gerbrands response key was used. The key was transilluminated by 7-W, 28-V bulbs. Keylight colors such as blue, red, green, and orange were used. Reinforcers were 3-s access to a raised hopper containing Purina Nutri-Blend ${ }^{\mathrm{TM}}$ pellets. The hopper was located behind a 5-cm square aperture, $14 \mathrm{~cm}$ from the chamber door and $7 \mathrm{~cm}$ from the floor. During reinforcer deliveries, the aperture was lit by a 7-W, 28-V bulb. Two 7-W, 28-V bulbs affixed to the outside of the left wall and located $1 \mathrm{~cm}$ above the ceiling and $6 \mathrm{~cm}$ from the door provided general illumination. A ventilation fan and white-noise generator provided ventilation and masked extraneous sound. A computer running Med-PC IV® software controlled experimental procedures and recorded data. 


\section{Procedure}

Pigeons were exposed to a series of two-component multiple schedules. The first component of a session was determined quasi-randomly with the restriction that the first component could not be the same for more than three successive sessions. Thereafter, the components strictly alternated every $10 \mathrm{~min}$, and were separated from one another by a 10-s blackout of the chamber. The schedules in a given component were suspended when the other component was in effect. Sessions terminated with the completion of the sixth component. Table 1 shows the keylight colors associated with each component, and the keylight colors used as the correlated and uncorrelated signals (see below).

Each of the conditions described below was in effect for a minimum of 13 sessions and until the mean percentage of time allocated to pausing across the last ten sessions of a condition differed by no more than 5\% from the means of the first and last 5-day blocks within those ten sessions. Table 2 shows the order of conditions and number of sessions per condition for individual pigeons.

\section{Unsignaled Baseline}

A two-component multiple schedule in which no signals accompanied pause-dependent reinforcer availability defined the Unsignaled Baseline. A concurrent (VI 90-s) (tandem VT 85-s DRO 5-s) schedule was in effect in both multiple schedule components. These concurrent schedules operated separately and independently of one another. Under the VI schedule, pecks to the key were reinforced immediately after the scheduled interval lapsed, as long as the IRT was $<5 \mathrm{~s}$. During the concurrently operating tandem schedule, following the lapse of the VT interval, the first pause of $5 \mathrm{~s}$ in key pecking was reinforced. In the conditions described below, the same multiple schedule described here was in effect. 


\section{Full Signal}

In both multiple-schedule components, key pecking was reinforced according to a VI schedule, as in the baseline condition described above. In one of the multiple-schedule components, hereafter labeled the Correlated-Signal component, a signal (i.e., a key-color change) indicating the availability of reinforcement for the next 5-s pause in key pecking occurred at the end of the VT interval. The signal terminated only after the reinforcer for pausing was delivered, after which the standard key color, or the key color associated with the multiple schedule for that particular component, was reinstated. Key pecks during the stimulus change were eligible for reinforcement when the VI interval lapsed and the IRT was $<5 \mathrm{~s}$. In the other component, hereafter labeled the Uncorrelated-Signal component, a key-color (different color from the Correlated-Signal component) change occurred according to a yoked-time (YT) $x$-s schedule, yoked in location and duration to that of the key color change in the Correlated-Signal component. If the Uncorrelated-Signal component was the first component of the session, the key-color change was yoked to the time in component and the signal duration during the last Correlated-Signal component in the previous session. Thus, the key-color change in the Uncorrelated-Signal component was uncorrelated with either peck- or pause-dependent reinforcement. If a reinforcer for key pecking was delivered during this uncorrelated-stimulus change, the timer controlling the stimulus change was stopped and restarted at the end of the reinforcement cycle. When this timer elapsed, the key color reverted back to the standard color (see Table 1).

\section{Brief Signal}

The multiple schedule that operated in the Brief Signal condition was identical to that described above for the Full-Signal component except for the duration of the signal. In the 
Correlated-Signal component, the signal reverted back to the standard component color $.5 \mathrm{~s}$ after its onset. Pecks that occurred prior to the lapse of $5 \mathrm{~s}$ reset the 5-s interval, at which point the signal was re-presented for the first $.5 \mathrm{~s}$. This peck-reset cycle continued until the pause requirement was met. As in the previous condition, in the Uncorrelated-Signal component, the .5 -s key color change was yoked in duration and location to the $.5 \mathrm{~s}$ stimulus changes that occurred in the preceding Correlated-Signal component.

\section{Other Procedural Details}

Sessions began with a 180-s blackout of the chamber. Because pigeons had experimental histories, neither magazine training nor key-peck shaping was necessary. The VI and VT schedules were composed of 15 intervals derived from Flesher and Hoffman's (1962) constantprobability algorithm. Sessions generally occurred at the same time every day and lasted for 60 minutes, excluding time for reinforcement.

\section{Results and Discussion}

Figure 1 shows the percentage of session time allocated to pausing for the last ten days of each condition for individual pigeons. The percentage of session time allocated to pausing was computed by summing all durations of pausing $\geq 5 \mathrm{~s}$ and dividing by component time in minutes, excluding reinforcement time, multiplied by 100. During the Unsignaled Baseline, time allocated to pausing did not differ systematically between the two components. Some between-subject variability, however, was evident. Pigeons 1022 and 1020 generally allocated more time to pausing than did Pigeons 942 and 1143. During Full- and Brief-Signal conditions, time allocated to pausing decreased from Unsignaled-Baseline levels in the Correlated-Signal component, whereas time allocated to pausing in the Uncorrelated-Signal component remained unchanged from Unsignaled-Baseline levels. Thus, the percentage of session time allocated to pausing was 
lower in the Correlated-Signal components than it was in the Uncorrelated-Signal components. This remained the case throughout the duration of each of the Full- and Brief-Signal conditions for each pigeon. No systematic difference in time allocation occurred between the Full- and Brief-Signal conditions, with Pigeon 1022 and 1143 showing relatively little variability and much overlap across the two conditions, and Pigeon 942 and 1020 showing a slight downward trend in time allocation across these conditions.

Figure 2 shows response (key peck) rates for the last ten days of each condition for individual pigeons. Response rate was calculated by dividing the total responses in a component by component time in minutes, excluding reinforcement time. Response rates, like time allocation, did not systematically differ across the two multiple-schedule components during the Unsignaled Baseline. When signals were present, response rates in the Correlated-Signal components generally were higher than in either the Unsignaled Baseline or the UncorrelatedSignal components. Response rates in the Uncorrelated-Signal components remained relatively similar to those in the Unsignaled Baseline throughout the experiment.

Figure 3 shows the mean (and standard deviation) reinforcement rates for VI (left panel) and DRO reinforcers (right panel) for a total of 20 sessions of the Unsignaled Baseline and Correlated-Signal and Uncorrelated-Signal components for Full- and Brief-Signal conditions. Reinforcement rates were calculated by dividing the total reinforcers per component, separated by source (i.e., VI or DRO), by component time, excluding reinforcement time. Generally, VI and DRO reinforcement rates did not differ across Correlated- and Uncorrelated-Signal components, nor did they differ across conditions.

To investigate the effects of signaling reinforcer availability on response patterns, logsurvivor analyses (Shull, Gaynor, \& Grimes, 2002; Shull, Grimes, \& Bennett, 2004) were 
conducted. Log-survivor analyses show the log proportion of total IRTs (defined as the time between two consecutive responses, eliminating postreinforcement pauses) on the y-axis that are greater than each elapsed time, or $t$, since the last response on the x-axis. Figure 4 shows these log-survivor analyses of IRTs for the last ten sessions of each condition for individual pigeons. The IRTs were pooled across replications of conditions, and, therefore, each data path represents data from 20 days for each condition. The left panel shows log-survivor plots from the Unsignaled Baseline, during which the slopes of log-survivor plots were similar across components and were relatively shallow. The right panel shows log-survivor plots from the Fulland Brief-Signaled conditions. In the Correlated-Signal component, the slopes of the logsurvivor plots generally were steeper than those in Unsignaled Baseline and those in the Uncorrelated-Signal component. The exception was Pigeon 942, which showed little difference between unsignaled and signaled conditions. As with time allocation and response rates, logsurvivor plots did not substantially differ between Full- and Brief-Signal conditions. The logsurvivor analyses show that responding in the Unsignaled Baseline and Uncorrelated-Signal component tended to occur in bouts, with periods of pausing followed by high rates of pecking. In the Correlated-Signal component, by contrast, pecking tended to occur at a relatively even rate, as evidenced by the steeper slope in comparison to the other conditions.

In Experiment 1, both key pecking and pausing were controlled by the contingencies imposed on the two respective operants. Pauses of the required duration and pecking under a VI schedule were assimilated into one another, yet both key pecking and pausing were controlled by the two contingencies concurrently in effect. Despite the assimilation of these additional long pauses in VI- maintained responding, reinforcement rates from the two sources of reinforcement 
remained approximately the same across all conditions and approximated the programmed rates; thus, the reinforcement of pausing did not disrupt the reinforcement of pecking, and vice versa.

Facilitated by signaling pause-dependent reinforcer availability, response rates increased for 3 of 4 pigeons under both Full-and Brief-Signal conditions in the Correlated-Signal component, relative to the Unsignaled Baseline. Additionally, log-survivor analyses reveal that key pecking in the Correlated-Signal component was more likely to occur at a relatively even rate. In contrast, key pecking in the Uncorrelated-Signal component remained similar to Unsignaled Baseline, in which it was more likely for pauses $\geq 5 \mathrm{~s}$ to be followed by bouts of pecking.

Signals correlated with pause-dependent reinforcer availability reduced time allocated to pausing relative to unsignaled conditions and conditions under which a signal was independent of reinforcer availability. Thus, the inclusion of the signal reduced pausing at times other than when a reinforcer was scheduled for a pause, making it more likely that pecking would be followed by shorter pauses in the presence of the standard stimulus. This observation is also substantiated by the differences in slope in log-survivor plots, which show pecking occurred at a more even rate in the Correlated-Signal component than the Uncorrelated-Signal component and the Unsignaled Baseline The discriminative control of pausing, in the Correlated-Signal component only, was demonstrated in the presence of both the brief and full signal, in that pauses $\geq 5$ s were less likely to occur outside of signaling pause-dependent reinforcer availability. No systematic difference across the behavioral measures (e.g., time allocation, response rates, response patterns) occurred between signals corresponding to less than the full duration of the pause requirement to conditions in which the entire pause requirement was signaled. 
Although the effects of signaling pause-dependent reinforcer availability produced similar effects on pigeons' time allocated to pausing, signaling had little effect on the response rates and response patterns for Pigeon 942. Craig et al. (2014) found that response rates were similar in both the DRO-Signal (correlated) and Arbitrary-Signal (uncorrelated) components for 2 of 3 pigeons, and in this regard, the response-rate results of Pigeon 942 are consistent with previous findings. Craig et al., however, found clear differences in the response patterns for 3 of 3 pigeons, and in this respect, the results of Pigeon 942 were inconsistent with both those of Craig et al. and the other pigeons in the current experiment. Because Pigeon 942 showed the least differentiation in time allocated to pausing between the Correlated- and UncorrelatedSignal components, it is possible that changing the pause requirement (e.g., increasing from 5 to 10 s) may have facilitated changes to response rate and pattern that were consistent with the other pigeons.

In this experiment, as in previous ones investigating pausing and pecking as concurrent operants, the discrete response was maintained by an interval schedule. Here, that schedule was VI, but as noted earlier, FI schedules also have been used. The second experiment investigated the control of pausing and pecking when the latter was maintained by a DRL schedule.

\section{Experiment 2}

Although FI schedules generate pauses, pausing is not a requirement of the reinforcement contingency. Under DRL schedules, pausing also occurs, but is a part of what is required for reinforcement, that is, the reinforcement contingency. If pausing is an operant, antecedent and consequent events should control it under different concurrent reinforcement conditions, not only interval schedules. Experiment 2 examined this possibility, as well as the generality of the discriminative control of pausing studied in Experiment 1. 


\section{Method}

\section{Subjects}

Four male White Carneau pigeons, different from those in Experiment 1 and each with a history of responding on different reinforcement schedules, were used. Each was housed separately in a vivarium with a 12:12-hr light/dark cycle and with continuous access to water and health grit in their home cages. Each was maintained at $80 \%$ of its ad libitum body weight (+/$15 \mathrm{~g})$ by use of postsession feedings.

\section{Apparatus}

The apparatus was similar to that described in Experiment 1, except that the work area was $32 \mathrm{~cm} \mathrm{Hx} 27 \mathrm{~cm} \mathrm{~W} \mathrm{x} 31 \mathrm{~cm} \mathrm{~L}$ and the key was $2-\mathrm{cm}$ in diameter.

\section{Procedure}

The procedure was similar to that of Experiment 1, but DRL schedules were used as the schedule of reinforcement for pecking rather than VI schedules. Key pecking first was reinforced according to a multiple DRL 15-s DRL 15-s schedule in which interresponse times (IRTs) $\geq 15 \mathrm{~s}$ were reinforced, and IRTs less than this reset the interreinforcer interval. After several sessions of exposure to the latter schedule, the DRL value was adjusted for each pigeon. The final values used were 25 s for Pigeon 14431, 20 s for Pigeon 14507 and Pigeon 14167, and 15 s for Pigeon 19841. During Unsignaled Baseline, a concurrent (DRL $x$ s) (tandem VT $55 \mathrm{~s}$ DRO $5 \mathrm{~s}$ ) was in effect in both multiple schedule components, where $x$ was set to the values described in the preceding sentence. Under the DRL $x$-s schedule, pecks that were spaced at least $x \mathrm{~s}$ apart were reinforced. In addition, the first pause $\geq 5 \mathrm{~s}$, after a variable amount of time (averaging $55 \mathrm{~s}$ ) elapsed, was reinforced. 
Each condition lasted a minimum of 13 days and was changed only when the mean percentage of time allocated to pausing across the last ten sessions of a condition differed by no more than 5\% from the means of the first and last 5- day blocks within those ten sessions. Table 3 shows the standard and signal keylight colors, as defined in Experiment 1, used for individual pigeons. Table 4 shows the order of conditions and number of sessions per condition for individual pigeons.

\section{Full Signal and Brief Signal}

The signal conditions and control conditions were as described in Experiment 1. As in Experiment 1, signals of different durations (i.e., Full and Brief) either signaled pause-dependent reinforcer availability in what again will be labeled the Correlated-Signal component, or were uncorrelated with such reinforcer availability in the Uncorrelated-Signal component.

\section{Results and Discussion}

Figure 5 shows the percentage of session time allocated to pausing during the last ten sessions of each condition for individual pigeons. These data were computed as described in Experiment 1. Because time allocation was restricted above $75 \%$, the y-axis begins at $50 \%$. In the Unsignaled-Baseline condition, time allocated to pausing did not systematically differ between the two components. During Full- and Brief-Signal conditions, time allocated to pausing decreased from Unsignaled-Baseline levels in the Correlated-Signal component, whereas time allocated to pausing in the Uncorrelated-Signal component remained near Unsignaled-Baseline levels. Thus, the percentage of overall session time allocated to pausing was lower in the Correlated-Signal component than the Uncorrelated-Signal component. This remained the case throughout replications of both the Full-and Brief-Signaled conditions, until returning to the 
Unsignaled Baseline. No systematic differences were observed between the Full- and BriefSignal conditions.

Figure 6 shows response (key peck) rates for the last ten days of each condition for individual pigeons. These data were computed as described in Experiment 1. Response rates, like time allocation, did not systematically differ across components during Unsignaled Baseline. During Full- and Brief-Signal conditions, response rates in the Correlated-Signal component generally were higher than the Unsignaled Baseline and the Uncorrelated-Signal component. For all pigeons, response rates in the Uncorrelated-Signal conditions remained relatively similar to Unsignaled-Baseline response rates throughout the experiment.

Figure 7 shows the mean (and standard deviation) reinforcement rate for DRL and DRO reinforcers delivered during the Unsignaled-Baseline conditions, and Correlated-Signal and Uncorrelated Signal-components during the Full- and Brief-Signal conditions. These data were computed as described in Experiment 1. Although there were no differences in DROreinforcement rates across conditions and components, some differences occurred in DRLreinforcement rates between the Correlated- and Uncorrelated-Signal Conditions. For 2 of 4 pigeons, reinforcement rates were higher slightly in the Uncorrelated-Signal components than the Correlated-Signal components, but this was perhaps influenced by preexisting baseline reinforcement rate differences.

Figure 8 shows the log-survivor analyses of IRTs (defined and calculated as in Experiment 1) from the last ten sessions of each condition for individual pigeons. The left panel shows log-survivor plots from the Unsignaled Baseline, during which plots were similar across components and the slopes were relatively shallow. The right panel shows log-survivor plots from the Full-and Brief-Signal conditions. The slopes of the log-survivor plots from the 
Correlated-Signal components were slightly steeper than those in Unsignaled Baseline and those in the Uncorrelated-Signal component. Overall, like time allocation and response rates, logsurvivor plots did not substantially differ between Full- and Brief-Signal conditions.

In Experiment 2, operant pausing and pecking maintained under a schedule that already required a pause in pecking were assimilated into one another. Although pauses of the required duration were embedded in the DRL- maintained responding, both key pecking and pausing were controlled by the two contingencies concurrently in effect. The additional pause requirement in the Unsignaled Baseline, however, was not correlated with what might be called optimal DRL performance under this schedule, namely a response rate that would allow the maximum reinforcement rates of 4 (DRL 15) or 2.4 (DRL 25) reinforcers per minute.

Under both Full-and Brief-Signal conditions, response rates increased in the CorrelatedSignal component, relative to the Unsignaled Baseline. Log-survivor analyses show that key pecking occurred at a relatively even and higher rate in the Correlated-Signal components under Full- and Brief-Signal conditions than in the Unsignaled-Baseline and Uncorrelated-Signal components. Bouts of responding, however, were only slightly different than those present in the Uncorrelated-Signal component and Unsignaled Baseline, likely as a function of the peck-pause reinforcement under DRL schedules. Increased response rates under a DRL schedule can reduce reinforcement rates, and in this manner, signals in the Correlated-Signal component did not necessarily making pecking more efficient. DRL-reinforcement rates, however, did not differ substantially across components or conditions for 2 of 4 pigeons, but were marginally higher in the Uncorrelated-Signal components for the other 2 pigeons. The largest difference in DRLreinforcement rates between the Correlated- and Uncorrelated-Signal components occurred for 
Pigeon 19841, although these differences between components were minimal (3-4 reinforcers per session) and appear to reflect differences present during the Unsignaled Baseline.

Signals correlated with pause-dependent reinforcer availability reduced time allocated to pausing relative to unsignaled conditions and conditions under which a signal was independent of reinforcer availability. Thus, the signal gained discriminative control of pausing, separate from that required by the pecking contingency. Such control of pausing, in the Correlated-Signal component only, was demonstrated by both the brief and full signal, in that pauses $\geq 5 \mathrm{~s}$ were less likely to occur outside of signaling pause-dependent reinforcer availability. No systematic differences across the behavioral measures (i.e., time allocation, response rates, response patterns) occurred between signals corresponding to less than the full duration of the pause requirement to conditions in which the entire pause requirement was signaled.

The results of Experiment 2 systematically replicated those of Experiment 1. As compared to Experiment 1, time allocated to pausing was higher in both components during the Unsignaled Baseline, likely as a function of the peck-pause reinforcement under DRL schedules arranged in Experiment 2. Overall, differences in time allocation, response rate, and peck-pause patterns between the Correlated- and Uncorrelated-Signal component were consistent with, but generally not as pronounced, as those in Experiment 1. Aside from schedule differences, it is important to note that comparisons made between Experiments 1 and 2 are between-subject comparisons; thus, individual variably may account for some of these differences.

Although pausing was explicitly reinforced under DRL schedules in Experiment 2, the discriminative control of pausing under DRO schedules still occurred. Thus, even under conditions in which pauses were embedded in another concurrently reinforced operant, pausing in DRO schedules came under discriminative control, thereby demonstrating the control of both 
operants. Beyond requiring the passage of a particular amount of time, some schedules allow for reinforcement rate to be maximized by increases in response rate alone. The third experiment investigated the control of pausing and pecking when the latter was maintained by an FR schedule.

\section{Experiment 3}

Like FI schedules, FR schedules generate postreinforcement pauses, but the two schedules control characteristically different response patterns. Under FR schedules, there is a rapid transition to a high, steady response rate that continues until reinforcement, while in FI schedules the transition is more gradual, although terminal response rates (just prior to reinforcement) may be quite high; there is, in addition, some evidence that long term exposure to FI schedules yields FR-like break and run performance (Schneider, 1969). An important difference, however, is that pausing is often more compatible with FI schedules than FR schedules, as increased pausing in the latter may be accompanied by reductions in reinforcement rate. Experiment 3 was designed to examined the control of pausing and pecking under conditions in which pecking was concurrently reinforced under FR schedules, as well as the generality of the discriminative control of pausing studied in Experiment 1 and 2.

\section{Method}

\section{Subjects}

Two male White Carneau pigeons from Experiment 2 were used.

\section{Apparatus}

The apparatus was as described in Experiment 2. 


\section{Procedure}

The procedure was similar to that of Experiment 1, but instead of VI schedules, FR 50 schedules were used to reinforce pecking. During Unsignaled Baseline, a concurrent (FR 50) (tandem VT 85 s DRO 5 s) was in effect in both multiple schedule components. Under the FR 50 schedule, 50 pecks were required for reinforcement. In addition, the first pause $\geq 5 \mathrm{~s}$ after a variable amount of time (averaging $85 \mathrm{~s}$ ) elapsed was reinforced. Following baseline, the FullSignal condition, as described in Experiment 1, was implemented, and was followed by a return to the Unsignaled Baseline. Table 5 shows the standard and signal keylight colors, as defined in Experiment 1, used for individual pigeons.

Each condition was in effect for a minimum of 13 days and was changed only when the mean percentage of time allocated to pausing across the last ten sessions of a condition differed by no more than $5 \%$ from the means of the first and last 5-day blocks within those ten sessions. Session length was 40 minutes rather than 60 minutes to avoid possible satiation. Table 6 shows the order of conditions and number of sessions per condition for individual pigeons.

\section{Results and Discussion}

Figure 9 shows the percentage of session time allocated to pausing during the last ten sessions of each condition for individual pigeons. These data were computed as described in Experiment 1. During Unsignaled Baseline, time allocated to pausing did not systematically differ between the two components. During the Full-Signal condition, time allocated to pausing decreased from Unsignaled-Baseline levels in the Correlated-Signal component, whereas time allocated to pausing in the Uncorrelated-Signal component remained near Unsignaled-Baseline levels. Thus, the percentage of overall session time allocated to pausing was lower in the 
Correlated-Signal component than the Uncorrelated-Signaled component. This remained the case until returning to the Unsignaled Baseline.

Figure 10 shows response (key peck) rates for the last ten days of each condition for individual pigeons. These data were computed as described in Experiment 1. Response rates, like time allocation, did not systematically differ across components during Unsignaled Baseline. During the Full-Signal condition, response rates in the Correlated-Signal component were higher than the Unsignaled-Baseline conditions and the Uncorrelated-Signal component. For both pigeons, response rates in the Uncorrelated-Signal component remained relatively similar to Unsignaled-Baseline response rates.

As FR schedules characteristically produce break-and-run performance, run rates and postreinforcement pauses were also analyzed. Figure 11 shows run rates, or the rate of responding following the postreinforcement pause, following FR reinforcers only. Run rates were calculated by dividing the number of responses made after the postreinforcement pause by the time between the first and last response of the ratio. Similar to overall response rates, run rates did not systematically differ across components during Unsignaled Baseline. Following the introduction of the Full-Signal condition, run rates between postreinforcement pauses in the Correlated-Signal component generally were higher than Unsignaled Baseline and the Uncorrelated-Signal component. For both pigeons, response rates in the Uncorrelated-Signal component remained relatively similar to Unsignaled-Baseline response rates. Figure 12 shows the mean postreinforcement pauses, or the period between FR reinforcer delivery until the next key peck, from the last ten sessions of each condition for individual pigeons. No systematic difference in postreinforcement pauses occurred between the two components during the Unsignaled-Baseline or Full-Signal conditions. 
Figure 13 shows the mean (and standard deviation) reinforcement rate for FR (left panel) and DRO (right panel) reinforcers for Correlated-Signal and Uncorrelated Signal-components in each condition. These data were computed as described in Experiment 1. The FR and DRO reinforcement rates did not differ substantially across components during Unsignaled Baseline, nor in the Full-Signal condition.

Figure 14 shows the log-survivor analyses of IRTs (defined as in Experiment 1) from the last ten sessions of each condition for individual pigeons. The left panel shows log-survivor plots from the Unsignaled Baseline, during which plots were similar across components, and the slopes were relatively shallow. The right panel shows log-survivor plots from the Correlated and Uncorrelated-Signal components. In the Correlated-Signal component, the slopes of the logsurvivor plots were slightly steeper than those in the Uncorrelated-Signal component.

In Experiment 3, operant pausing and FR-maintained responding were assimilated into one another, under which key pecking and pausing were controlled by the two contingencies concurrently in effect. Although pausing already was characteristic of the FR schedule (but not required) in place for pecking (i.e., postreinforcement pause), both key pecking and pausing were controlled by the two contingencies concurrently in effect. Despite FR reinforcement for pecking, reinforcement rates for pausing approximated the programmed rates; thus, the inclusion of FR schedules did not disrupt the reinforcement of pausing.

Signals correlated with pause-dependent reinforcement availability reduced time allocated to pausing relative to unsignaled conditions and conditions where a signal was independent of such reinforcer availability. Facilitated by the signaling of reinforcer availability for pausing, response rates increased in the Correlated-Signal component, relative to Unsignaled Baseline, under the Full-Signal condition. Similar to the effects on response rate, signaling such 
reinforcer availability increased run rates to a greater extent in the Correlated-Signal condition than in the Uncorrelated-Signal condition. Despite these increases in response and run rates, the mean postreinforcement pause did not increase in the Correlated-Signal component from the Unsignaled Baseline. Thus, increases in run rates show that pauses $\geq 5$ s occurred less frequently during ratio runs and that pausing was more likely to be assimilated into the postreinforcement pause. Despite the aforementioned differences in pausing and pecking, reinforcement rates from the two sources of reinforcement rates did not differ substantially across components during Unsignaled Baseline, nor in the Full-Signal condition.

The results of Experiment 3 systematically replicated those of Experiments 1 and 2, but with key-peck responding maintained by an FR 50 schedule. A systematic replication of Experiment 2 was demonstrated within-subject, as two of the same pigeons from Experiment 2 were used in Experiment 3. Differences in time allocated to pausing were consistent with, but generally not as pronounced, as those in Experiment 1, and were lower throughout conditions compared to Experiment 2. These differences were likely a function of the schedule under which pecking was reinforced. Under FR schedules, a rapid transition to a high, steady response rate continues until reinforcement, and, thus, more time allocated to pausing may be accompanied by reductions in reinforcement rate. As compared to Experiments 1 and 2, rather than interrupting pecking, pausing was more likely to be assimilated into the postreinforcement pause in Experiment 3.

\section{General Discussion}

In a series of three experiments, the relation between the schedule of reinforcement arranged for pecking (e.g., VI, DRL and FR schedules) and the duration of the antecedent discriminative stimulus (i.e., full or brief signal) to the discriminative control of operant pausing 
was analyzed. In each experiment, pauses $\geq 5 \mathrm{~s}$ were less likely to occur when a key-color change signaled the availability of a pause-dependent reinforcer than if the signal was either absent or uncorrelated with pause-dependent reinforcers. Additionally, signaling such availability generally affected the rate of pecking. In Correlated-Signal components, response rates increased relative to the Unsignaled Baseline. In Uncorrelated-Signal components, response rates were unchanged from the Unsignaled Baseline. The results of Experiment 1 replicate previous research (Craig et al., 2014; Lattal \& Boyer, 1980; Rachlin \& Baum, 1972; Zeiler, 1976; 1977) showing the control of both pausing and pecking under interval schedules of reinforcement. The results of Experiments 2 and 3 show how pausing as an operant melds with a discrete operant response when the latter is controlled by DRL (Experiment 2) or FR (Experiment 3) schedules.

Signaling reinforcer availability for pausing decreased time allocated to pausing and increased response rates relative to conditions where the signal was either absent or uncorrelated with reinforcement availability. Duration of the signal, whether brief or encompassing the entire pause requirement, made no systematic difference on any of the behavioral measures, replicating and extending the findings of Schaal and Branch (1988).

The five issues most germane to the results of the three experiments reported herein are: the nature of pausing as an operant, pausing in the context of the reinforcement of a discrete response, signaling reinforcer availability for pausing, pausing and the Matching Law, and potential applications to problems of social significance. These issues are discussed in the sections that follow. 


\section{Pausing as an operant}

Pausing is a rather generic concept, negatively defined in that it must be defined relative to something else, be it behaving in general or a particular discrete response class, like pecking. The use of DRO schedules arranges for the explicit reinforcement of pausing, in that nonengagement in the target response is required for a specified amount of time. When pausing is reinforced concurrently with another targeted, discrete-operant response, however, any discussion of pausing must also specify the parameters of reinforcement maintaining the discrete response.

Lattal and Boyer's (1980) explicit definition of pausing as a period $\geq 5 \mathrm{~s}$ of nonengagment in key pecking emerged from pilot data demonstrating that shorter pauses during the FI 150-s baseline occurred very frequently (e.g., $1 \mathrm{~s}$ ) and longer pauses (e.g., $10 \mathrm{~s}$ ) occurred so infrequently that, under both conditions, there was concern that these pauses would not be sensitive to experimental manipulations. These parameters, however, should not be interpreted as absolute durations. Because pausing is defined relative to a discrete response, the extent to which the schedule of reinforcement for the discrete response allows for the assimilation of pauses of a particular duration must be considered.

Among these definitional considerations is some question as to what the "pausing operant" was in this series of experiments. Although that operant was defined as "pausing" in key pecking, it also is possible that "other" behavior increased as a function of signaling its reinforcer availability. Anecdotal observations of pigeons during several sessions revealed that they tended to spin around, preen, and peck other regions in the operant chamber and panel aside from the key following signal changes. Therefore, it is possible that "other" behavior like the above was reinforced. This possibility would be best substantiated using systematic observations 
(see Skinner, 1948; Staddon, 1992) showing that this class of "other" responses has been reinforced. Without such observations, it is perhaps more parsimonious and useful to discuss the current results in terms of the programmed dependency, and subsequently, the decrease in a measured nonengagement response rather than assumptions based on increases in "other" responses.

\section{Pausing in the context of the reinforcement of a discrete response}

Unsignaled-Baseline performance, relative to which the effects of signals were subsequently evaluated, differed across experiments as a function of the schedule of reinforcement arranged for pecking. Important to note is that the evaluation of some of these differences are based on between-subject comparisons, and, as such, are limited. In the presence of the concurrent DRO contingency, under VI schedules, pecking was more likely to occur in bouts, as evidenced by the log-survivor analyses.

Under DRL schedules, in Experiment 2, however, pecks were followed by pauses that were longer generally than those in Experiment 1, evidenced by the difference in slopes between log-survivor plots in Experiment 2 compared to those in Experiment 1. In addition to these differences in peck-pause patterns, the overall rate of pecking was lower during the Unsignaled Baseline in Experiment 2 than Experiment 1, and time allocated to pausing was higher in Experiment 2. The DRL schedule for pecking was itself a peck-pause requirement, and therefore, required pauses $\geq 15 \mathrm{~s}$ to maintain reinforcement for pecking. Thus, pauses of the required duration were assimilated into the DRL schedule. These findings are perhaps not entirely surprising, as the DRL requirement was at least $15 \mathrm{~s}$, and the required pause was only $5 \mathrm{~s}$. Thus, pauses of the required duration occurred regularly as a result of the reinforcement contingency for pecking (see also Lattal \& Boyer, 1980). Had the pause requirement in the DRL schedule 
been shorter in duration (e.g., $5 \mathrm{~s}$ ), pauses would approach or exceed the pause requirement present in the pecking contingency. Under these conditions, reinforcement under either the pecking or pausing contingency (or both) would be disrupted. Thus, it is still seems likely that parameters of a DRL schedule could alter the assimilation of the concurrently reinforced pausing.

As compared to Experiments 1 and 2, in the Unsignaled Baseline, pecks generally were followed by short pauses between each peck in Experiment 3, evidenced by the relatively steep slope of the log-survivor plots between 0 and $1 \mathrm{~s}$ elapsed times. These differences likely are attributable to the response-reinforcer relation present in FR schedules. Reinforcement rate in FR schedules is driven solely by response rate, and therefore, reinforcement rates can be maximized by responding at higher rates (and pausing for short durations). Analysis of run rates in Experiment 3 suggest that responding during the FR runs often was not disrupted once the ratio run had started. Additionally, pauses of the required duration were assimilated into the postreinforcement pauses without increasing the duration of the average postreinforcement pause. Postreinforcement pauses are at least partially a function of the ratio size (e.g., Felton \& Lyon, 1966; Powell, 1968). For example, an FR 5 should produce a shorter postreinforcement pause than an FR 50, which may be less compatible with the required pause duration. Parameters of the FR schedule, therefore, still may modulate the assimilation of the concurrent reinforcement of pausing.

\section{Signaling reinforcer availability for pausing}

The results of all three experiments systematically replicated the results of Craig et al. (2014), and are consistent with those of signaling reinforcer availability for a discrete response (Marcucella, 1976; Marcucella \& Margolius, 1978), in which pecking was reduced to near-zero 
rates outside of signals corresponding with its reinforcer availability. In the current experiments, however, pauses of the required duration occurred occasionally outside of the signal (see also Craig et al., 2014). Across experiments, log-survivor analyses show that pauses $20 \mathrm{~s}$ or longer occurred at least occasionally for all pigeons during in the Correlated-Signal component under both the Full- and Brief-Signal conditions, even though reinforcement of pausing in the Correlated-Signal component never occurred outside of the signals. During Experiment 1, this was likely a function of the VI contingency in place for pecking, under which pauses may alternate naturally with pecking (see Staddon \& Simmelhag, 1970). In Experiment 2, this observation is perhaps not surprising, as pauses of the required duration were assimilated into the DRL requirement, which required pauses of at least $15 \mathrm{~s}$. Finally, time allocated to pausing was captured in the postreinforcement pauses present in FR schedules in Experiment 3. Thus, although antecedent discriminative control was not perfect, it was more probable that pauses of the required duration would occur in the presence of signals corresponding to pause-dependent reinforcer availability.

Nearly identical discriminative control of pausing under both a signal that spanned the duration of the pause required for reinforcement and one that occurred only briefly when a pause-dependent reinforcer became available and then disappeared was observed across various behavioral measures (i.e., time allocation, response rate, response pattern). This similarity replicated and extended Schaal and Branch's (1988) finding of no difference between a brief and full signal in the maintenance of key-pecking when reinforcement was delayed. It was not until 27-s delays were imposed that Schaal and Branch observed differences between the two different signal durations. In the current experiments, only two signal durations were investigated. It is possible that signals $<.5 \mathrm{~s}$ in duration, or between $.5 \mathrm{~s}$ and the full pause requirement, would 
differentially control pausing. Furthermore, there is some question as to whether it is the absolute duration of the signal or its duration relative to the delay or pause requirement in effect that is the critical feature. Although keylight color changes served as the signal in the current experiments, several variations in the presentation of the signal, such as superimposing a shape or line on the key (cf. Weisman, 1970), and other stimulus modalities (e.g., a tone) warrant attention.

In addition to the parameters of the signal, parameters of the correlation between the signal and reinforcement may also modulate effectiveness of the signal in controlling pausing. In the current experiments, the correlation between the signal and reinforcer availability was 1 in the Correlated-Signal component, and functionally 0 in the Uncorrelated-Signal component. It is unknown whether signal correlations greater than 0 but less than 1 would differentially control pausing, similar to the effects observed with discrete responding (Marcucella, 1976).

Additionally, the reinforcement contingency in place for pausing was an FR 1, as only one continuous pause $\geq 5 \mathrm{~s}$ was required for reinforcement (after the passage of a variable amount of time). The effects of including, for example, an FR 5 schedule of reinforcement for pausing (i.e., five pauses of the required duration must occur prior to the reinforcer becoming available), and signaling the availability of such reinforcers, are unknown.

\section{Pausing and the Matching Law}

Herrnstein (1970) noted the importance of considering “other" sources of reinforcement for "other" responses by including the variable $\mathrm{r}_{\mathrm{o}}$ in the matching law. It is generally agreed that $r_{o}$ measures responses that are controlled by background sources of reinforcement (Herrnstein, 1970; 1974; Heyman \& Monaghan, 1987). Although the particular value of $r_{o}$ may change as a function of operations that directly affect these "other" sources of reinforcement, the value of $r_{o}$ may also change as a function of changes made to the source of operant reinforcement. For 
example, changes in the arranged reinforcer magnitude, reinforcer quality, or organism's deprivation level may all lead to subsequent changes in the $r_{o}$ parameter (de Villiers \& Herrnstein, 1976; Heyman \& Monaghan, 1987). Relatively few studies have directly manipulated these background sources of reinforcement (see Belke \& Heyman, 1994). Therefore, much of the work surrounding $\mathrm{r}_{\mathrm{o}}$ involves the inference of changes in "other" behavior as a result of changes in the operant source of reinforcement.

The current series of experiments explicitly arranged for reinforcement of such "other" behavior, providing an experimental analysis of "other" behavior rather than a conceptual analysis. Despite reinforcing such "other" behavior, the current experiments arranged for the reinforcement of pausing in a way most similar to that used in experiments where concurrently available topographically different operants have been reinforced (e.g., McSweeney, 1978; Sumpter et al., 1998). Although the reinforcement of topographically different operants most often involves different discrete responses (e.g., key pecking and door pushing), topographically different variations of the same targeted response (e.g., pecking and pausing in pecking) were reinforced in the current series of experiments. In the current experiment, however, one response was discrete (pecking) and the other was continuous (pausing) in nature. Despite such differences, key pecking under both of these arrangements was affected by the parameters of a concurrently reinforced and topographically different response.

Although "other" behavior may have been captured in the reinforcement of pausing, reinforcers other than the programmed reinforcer (e.g., 3-s access to food pellets) perhaps were available still for these "other" responses. It is important to note, therefore, that $r_{o}$ is not isomorphic with the reinforcement of "other" behavior. It is likely that $r_{o}$ was reduced as a function of directly reinforcing pausing, as manipulations to operant sources of reinforcement 
affect $r_{o}$ (de Villiers \& Herrnstein, 1976; Heyman \& Monaghan, 1987), but it is possible that it was not eliminated entirely. For example, the reinforcers associated with preening presumably were available still throughout experimental sessions, and as such, may have exerted some control over such "other" behavior.

\section{Potential Applications}

Most applied research using DRO contingencies has focused on the elimination of behavior (see Poling \& Ryan, 1982; Vollmer \& Iwata, 1992). However, reinforcement of both engagement and nonengagement in a target response may occur frequently outside of the laboratory as a result of either failures in treatment integrity or the inability to completely discontinue reinforcer delivery for problem behavior. Therefore, the interactive effects that were demonstrated in the current series of experiments may have implications for applied settings. The DRO arrangement used in the current series of experiments is most akin to a momentary-DRO schedule, in which reinforcement occurs if the response does not occur at the end of a fixed or variable interval (e.g., Powell et al., 1977; Lindberg et al., 1999). The current findings that signaled (both brief or full), pause-dependent reinforcement decreased time allocation to pausing is similar to findings in the applied literature with self-injurious behavior (see Hammond, Iwata, Fritz, \& Dempsey, 2011). There are, however, possible implications to problem behavior aside from self-injurious behavior. One of the most effective treatments for substance abuse is contingency management $(\mathrm{CM})$, which relies on providing vouchers to increase abstinence from drug use (see Lussier, Heil, Mongeon, Badger, \& Higgins, 2006 for review). "Abstinence” may be conceptualized as a period of pausing in drug use, and is most often probed after a period of time has elapsed (therefore, akin to a momentary-DRO arrangement). Thus, based on the current findings, the extent to which probes of abstinence are signaled (e.g., the establishing of regular 
appointment times or providing clear windows of time in which drugs can be detected via various biological tests) may predict the control of the reinforcers for pausing in the context of concurrently available reinforcers for responding (e.g., drug use).

\section{Conclusion}

The concept of the operant is central to the experimental analysis of behavior, yet, most often, operants have been investigated as discrete responses. The three experiments reported in this dissertation demonstrate not only how pausing in a targeted response functions as an operant (see also Craig et al., 2014; Lattal \& Boyer, 1980), but also this operant's sensitivity to both antecedent events and schedule context. Along with the experiments cited in the previous sentence, the present experiments offer a method for the further experimental analysis of pausing as an operant.

Many of the basic parameters of pausing as an operant, however, remain understudied relative to other operants. Future research might arrange for an investigation of additional parameters of the schedule of reinforcement for the discrete response, as it may modulate the operant control of pausing. Additionally, several parameters of the signal deserve future investigation, including signal duration, both relative and absolute; modality; and its correlation with predicting reinforcer availability. Finally, many issues regarding the relation between the $r_{o}$ concept and operant pausing remain and invite further consideration, including the presence of $r_{o}$ in arrangements explicitly reinforcing "other" behavior, as in the current series of experiments. 


\section{References}

Azzi, R., Fix, D. S. R., Keller, F. S., \& Rocha e Silva, M. I. (1965). Exteroceptive control of response under delayed reinforcement. Journal of the Experimental Analysis of Behavior, 7, 159-162. doi: /10.1901/jeab.1964.7-159

Baum, W. M. (1974). On two types of deviation from the matching law: Bias and undermatching. Journal of the Experimental Analysis of Behavior, 22, 231-242. doi: 10.1901/jeab.1974.22-231

Baum, W.M. (1979). Matching, undermatching, and overmatching in studies of choice. Journal of the Experimental Analysis of Behavior, 32, 269-281. doi: 10.1901/jeab.1979.32-269

Baum, W. M., \& Rachlin, H. C. (1969). Choice as time allocation. Journal of the Experimental Analysis of Behavior, 12, 861-874. doi: 10.1901/jeab.1969.12-861

Catania, A. C. (1963). Concurrent performances: Reinforcement interaction and response independence. Journal of the Experimental Analysis of Behavior, 6, 253-263. doi: 10.1901/jeab.1963.6-253

Catania, A. C. (1970). Reinforcement schedules and psychophysical judgments: A study of some temporal properties of behavior. In W. N. Schoenfeld (Ed.), Theory of reinforcement schedules. New York: Appleton-Century-Crofts, pp. 1-42.

Catania, A. C. (2007). Learning (4th interim Ed.). Cornwallon-Hudson, NY: Sloan Publishing

Catania A.C, \& Keller, K.J. (1981). Contingency, contiguity, correlation, and the concept of causation. In: Harzem P, Zeiler M.D, editors. Advances in analysis of behaviour: Vol. 2. Predictability, correlation, and contiguity. Chichester England: Wiley. pp. 125-167 
Craig, A. R., Lattal, K. A., \& Hall, E. G. (2014). Pausing as an operant: Choice and discriminated responding. Journal of the Experimental Analysis of Behavior, 101, 230245. doi: $10.1002 /$ jeab.73

de Villiers, P. A., \& Herrnstein, R. J. (1976). Toward a law of response strength. Psychological Bulletin, 83, 1131-1153. doi: 10.1037//0033-2909.83.6.1131

Felton, M., \& Lyon, D. O. (1966). The postreinforcement pause. Journal of the Experimental Analysis of Behavior, 9, 131-134. doi: 10.1901/jeab.1966.9-131

Findley, J. D. (1958). Preference and switching under concurrent scheduling. Journal of the Experimental Analysis of Behavior, 1, 123- 144. doi, 10.1901/jeab.1958.1-123

Fleshler, M., \& Hoffman, H. S. (1962). A progression for generating variable-interval schedules. Journal of the Experimental Analysis of Behavior, 5, 529-530. doi: 10.1901/jeab.1962.5529

Hammond, J. L., Iwata, B. A., Fritz, J. N., \& Dempsey, C. M. (2011). Evaluation of fixed momentary DRO schedules under signaled and unsignaled arrangements. Journal of Applied Behavior Analysis, 44, 69-81. doi: 10.1901/jaba.2011.44-69

Herrnstein, R. J. (1961). Relative and absolute strength or response as a function of frequency of reinforcement. Journal of the Experimental Analysis of Behavior, 4, 267-272. doi: 10.1901/jeab.1961.4-267

Herrnstein, R. J. (1970). On the law of effect. Journal of the Experimental Analysis of Behavior, 13, 243-266. doi: 10.1901/jeab.1970.13-243

Herrnstein, R. J. (1974). Formal properties of the matching law. Journal of the Experimental Analysis of Behavior, 21, 159-164. doi: 10.1901/jeab.1974.21-159 
Heyman, G. M., \& Monaghan, M. M. (1987). Effects of changes in response requirement and deprivation on the parameters of the matching law equation: New data and review. Journal of Experimental Psychology: Animal Behavior Processes, 13, 384-394. doi: 10.1037//0097-7403.13.4.384

Imam, A. A., \& Lattal, K. A. (1988). Effects of alternative reinforcement sources: A reevaluation. Journal of the Experimental Analysis of Behavior, 50, 261-271. doi: 10.1901/jeab.1988.50-261

Kelleher, R. T. (1961). Schedules of conditioned reinforcement during experimental extinction. Journal of the Experimental Analysis of Behavior, 4, 1-5. doi: 10.1901/jeab.1961.4-1

Kramer, T. J., \& Rilling, M. (1970). Differential reinforcement of low rates: A selective critique. Psychological Bulletin, 74, 225-254. doi: 10.1037/h0029813

Lane, H. (1961). Operant control of vocalizing in the chicken. Journal of the Experimental Analysis of Behavior, 4, 171-177. doi: 10.1901/jeab.1961.4-171

Lattal K. A. (1989). Contingencies on response rate and resistance to change. Learning and Motivation, 20, 191-203. doi: 10.1016/0023-9690(89)90017-9

Lattal, K. A. (1991). Scheduling positive reinforcers. In I. H. Iversen \& L. A. Lattal (Eds.), Experimental analysis of behavior (pp. 87-134). New York: Elsevier.

Lattal, K. A., \& Boyer, S. S. (1980). Alternative reinforcement effects on fixed-interval performance. Journal of the Experimental Analysis of Behavior, 34, 285-296. doi: 10.1901/jeab.1980.34-285

Lattal, K. A., \& Ziegler, D. R. (1982). Briefly delayed reinforcement: An interresponse time analysis. Journal of the Experimental Analysis of Behavior, 37, 407-416. doi: 10.1901/jeab.1982.37-407 
Lindberg, J. S., Iwata, B. A., Kahng, S., \& DeLeon, I. G. (1999). DRO contingencies: An analysis of variable-momentary schedules. Journal of Applied Behavior Analysis, 32, 123-136. doi: 10.1901/jaba.1999.32-123

Lussier J. P., Heil S. H., Mongeon J. A., Badger G. J., Higgins S. T. (2006). A meta-analysis of voucher-based reinforcement therapy for substance use disorders. Addiction, 101, 192203. doi: 10.1111/j.1360-0443.2006.01311.x

Marcucella, H. (1974). Signalled reinforcement in differential reinforcement of low-rateschedules. Journal of the Experimental Analysis of Behavior, 22, 381-390. doi: 10.1901/jeab.1974.22-381

Marcucella, H. (1976). Signalled reinforcement and multiple schedules. Journal of the Experimental Analysis of Behavior, 26, 199-206. doi: 10.1901/jeab.1976.26-199

Marcucella, H., \& Margolius, G. (1978). Time allocation in concurrent schedules: The effect of signaled reinforcement. Journal of the Experimental Analysis of Behavior, 29, 419-430. doi: $10.1901 /$ jeab.1978.29-419

Moffitt, M. and Shimp, C. P. (1971). Two-key concurrent paced variable-interval paced variableinterval schedules of reinforcement. Journal of the Experimental Analysis of Behavior, 16, 39-49. doi: 10.1901/jeab.1971.16-39

Nevin, J. A. (1968). Differential reinforcement and stimulus control of not responding. Journal of the Experimental Analysis of Behavior, 11, 715-726. doi: 10.1901/jeab.1968.11-715

Perone, M., \& Courtney, K. (1992). Fixed ratio pausing: Joint effects of past reinforcer magnitude and stimuli correlated with upcoming magnitude. Journal of the Experimental Analysis of Behavior, 57, 33-46. doi:10.1901/jeab.1992.57-33 
Pierce, C. H., Hanford, P. V., \& Zimmerman, J. (1972). Effects of different delay of reinforcement procedures on variable-interval responding. Journal of the Experimental Analysis of Behavior, 18, 141-146. doi:10.1901/jeab.1972.18-141

Poling, A., \& Ryan, C. (1982). Differential reinforcement of other behavior schedules: Therapeutic applications. Behavior Modification, 6, 3-21. doi: $10.1177 / 01454455820061001$

Powell, R. W. (1968). The effects of small sequential changes in fixed-ratio size upon the post-reinforcement pause. Journal of the Experimental Analysis of Behavior, 11, 589-593. doi: 10.1901/jeab.1968.11-589

Powell, R. W. (1969). The effect of reinforcement magnitude upon responding under fixed-ratio schedules. Journal of the Experimental Analysis of Behavior, 12, 605-608. doi: 10.1901/jeab.1969.12-605

Powell, J., Martindale, B., Kulp, S., Martindale, A., \& Bauman, R. (1977). Taking a closer look: Time sampling and measurement error. Journal of Applied Behavior Analysis, 10, 325332. doi:10.1901/jaba.1977.10-325

Rachlin, H., \& Baum, W. M. (1972). Effects of alternative reinforcement: Does the source matter? Journal of the Experimental Analysis of Behavior, 18, 231-241. doi: 10.1901/jeab.1972.18-231

Reynolds, G. S. (1961). Behavioral contrast. Journal of the Experimental Analysis of Behavior, 4, 57-71. doi:10.1901/jeab.1961.4-57

Richards, R. W. (1981). A comparison of signaled and unsignaled delay of reinforcement. Journal of the Experimental Analysis of Behavior, 35, 145-152. doi: 10.1901/jeab.1981.35-145 
Richards, J. B., Sabol, K. E., \& Seiden, L. S. (1993). DRL interresponse-time distributions: Quantification by peak deviation analysis. Journal of the Experimental Analysis of Behavior, 60, 361-385. doi:10.1901/jeab.1993.60-361

Schaal D.W, \& Branch M.N. (1988). Responding of pigeons under variable-interval schedules of unsignaled, briefly signaled, and completely signaled delays to reinforcement. Journal of the Experimental Analysis of Behavior, 50,33-54. doi:10.1901/jeab.1988.50-33

Schoenfeld, W. N., Farmer, J., \& Vickery, C. (1970). Effects of varying probability of a response-pause requirement on a regular reinforcement baseline. Psychonomic Science, 18, 177-179. doi:10.3758/bf03332363

Shimp, C. P. (1968). Magnitude and frequency of reinforcement and frequencies of interresponse times. Journal of the Experimental Analysis of Behavior, 11, 525-535. doi: 10.1901/jeab.1968.11-525

Shull, R. L., Gaynor, S. T., \& Grimes, J. A. (2002). Response rate viewed as engagement bouts: Resistance to extinction. Journal of the Experimental Analysis of Behavior, 77, 211-231. doi:10.1901/jeab.2002.77-211

Shull, R. L., Grimes, J. A., \& Bennett, J. A. (2004). Bouts of responding: The relation between bout rate and the rate of variable-interval reinforcement. Journal of the Experimental Analysis of Behavior, 18, 65-83. doi:10.1901/jeab.2004.81-65

Sizemore, O. J., \& Lattal, K. A. (1977). Dependency, temporal contiguity, and responseindependent reinforcement. Journal of the Experimental Analysis of Behavior, 27, 119125. doi:10.1901/jeab.1977.27-119

Skinner, B. F. (1935). Two types of conditioned reflex and a pseudo type Journal of General Psychology, 12, 66-77. doi:10.1080/00221309.1935.9920088 
Skinner, B. F. (1948). 'Superstition' in the pigeon. Journal of Experimental Psychology, 121, 3, 273-274. doi:10.1037/h0055873

Staddon, J. E. R. (1965). Some properties of spaced responding in pigeons. Journal of the Experimental Analysis of Behavior, 8, 19-27. doi:10.1901/jeab.1965.8-19

Staddon, J. E. R. (1992). The 'superstition' experiment: a reversible figure. Journal of Experimental Psychology: General, 121, 3, 270-272. doi: 10.1037//0096-3445.121.3.270

Sumpter, C., Temple, W., \& Foster, T. (1998). Response form, force, and number: Effects on concurrent-schedule performance. Journal of the Experimental Analysis of Behavior, 70, 45-68. doi:10.1901/jeab.1998.70-45

Topping, J. S., Larmi, O.K., \& Johnson, D. L. (1972). Omission training: Effects of gradual introduction. Psychonomic Science, 28, 279-280. doi: 10.3758/bf03328739

Uhl, C. N., \& Garcia, E. E. (1969). Comparison of omission with extinction in response elimination in rats. Journal of Comparative and Physiological Psychology, 69, 554-562. doi: $10.1037 / \mathrm{h} 0028243$

Vollmer, T. R., \& Iwata, B. A. (1992). Differential reinforcement as treatment for behavioral disorders: Procedural and functional variations. Research in Developmental Disabilities, 13, 393-417. doi: 10.1016/0891-4222(92)90013-v

Weisman, R. G. (1970). Factors influencing inhibitory stimulus control: Differential of other behavior during discrimination training. Journal of the Experimental Analysis of Behavior, 12, 229-237. doi:10.1901/jeab.1969.12-229

Williams, B. A. (1976). The effects of unsignaled delayed reinforcement. Journal of the Experimental Analysis of Behavior, 26, 441-449. doi:10.1901/jeab.1976.26-441 
Zeiler, M. D. (1971). Eliminating behavior with reinforcement. Journal of the Experimental Analysis of Behavior, 16, 401-405. doi:10.1901/jeab.1971.16-401

Zeiler, M. D. (1976). Positive reinforcement and the elimination of reinforced responses. Journal of the Experimental Analysis of Behavior, 26, 37-44. doi:10.1901/jeab.1976.26-37

Zeiler, M. D. (1977). Elimination of reinforced behavior: Intermittent schedules of notresponding. Journal of the Experimental Analysis of Behavior, 25, 23-32.doi: 10.1901/jeab.1977.27-23

Zeiler, M.D., \& Blakely, T.F. (1983). Choice between response units: The rate constancy model. Journal of the Experimental Analysis of Behavior, 39, 275-291. doi: 10.1901/jeab.1983.39-275

Zeiler, M. D. \& Fite, G. M. (1975). Reinforcement of responding and not responding: Alternative responses. Bulletin of the Psychonomic Society, 6, 276- 278. doi: $10.3758 / \mathrm{bf0} 03336660$ 
Table 1

Experiment 1 Stimuli. Standard color refers to the key color corresponding with the respective multiple schedule component and Signal color refers to the key color during the during either the 1) DRO portion of the chain VT DRO schedule or 2) stimulus change in the Uncorrelated-Signal component.

\begin{tabular}{|c|c|c|c|c|}
\hline \multirow[b]{3}{*}{ Pigeon } & \multicolumn{4}{|c|}{ Component } \\
\hline & \multicolumn{2}{|c|}{ Correlated Signal } & \multicolumn{2}{|c|}{ Uncorrelated Signal } \\
\hline & Standard Color & Signal Color & Standard Color & Signal Color \\
\hline 942 & Red & White & Green & Orange \\
\hline 1022 & Green & Orange & Red & White \\
\hline 1143 & Orange & White & Blue & Red \\
\hline 1020 & Blue & Red & Orange & White \\
\hline
\end{tabular}


Table 2

Experiment 1 order of conditions and number of sessions for individual pigeons. $U=$ Unsignaled-Baseline condition, $F=$ Full-Signal condition, B = Brief-Signal condition.

\begin{tabular}{lcc}
\hline Pigeon & Condition & Sessions \\
& & \\
\hline 942 & U & 81 \\
& B & 41 \\
& F & 16 \\
& B & 15 \\
& F & 18 \\
& U & 39 \\
\hline 1022 & U & 81 \\
& B & 50 \\
& F & 16 \\
& B & 27 \\
& F & 15 \\
1143 & U & 29 \\
& U & 88 \\
& F & 33 \\
& B & 30 \\
& F & 15 \\
& B & 19 \\
& U & 32 \\
\hline 1020 & U & 94 \\
& F & 25 \\
& B & 21 \\
& F & 16 \\
& B & 23 \\
& U & 42 \\
\hline
\end{tabular}


Table 3

Experiment 2 Stimuli. Standard color refers to the key color corresponding with the respective multiple schedule component and Signal color refers to the key color during the during either the 1) DRO portion of the chain VT DRO schedule or 2) stimulus change in the Uncorrelated-Signal component.

\section{Component}

Correlated Signal Uncorrelated Signal

\begin{tabular}{ccccc}
\hline Pigeon & Standard Color & Signal Color & Standard Color & Signal Color \\
\hline 14167 & White & Blue & Green & Red \\
14507 & Green & Red & White & Blue \\
19841 & Green & Red & White & Blue \\
14431 & White & Blue & Green & Red \\
\hline
\end{tabular}


Table 4

Experiment 2 order of conditions and number of sessions for individual pigeons. $U=$ Unsignaled-Baseline condition, $F=$ Full-Signal condition, $B=$ Brief-Signal condition .

\begin{tabular}{|c|c|c|}
\hline Pigeon & Condition & Sessions \\
\hline \multirow[t]{6}{*}{14167} & $\mathrm{U}$ & 95 \\
\hline & $\mathrm{F}$ & 24 \\
\hline & B & 30 \\
\hline & $\mathrm{F}$ & 30 \\
\hline & B & 28 \\
\hline & $\mathrm{U}$ & 15 \\
\hline \multirow[t]{6}{*}{14507} & $\mathrm{U}$ & 95 \\
\hline & $\mathrm{F}$ & 41 \\
\hline & B & 27 \\
\hline & $\mathrm{F}$ & 23 \\
\hline & B & 17 \\
\hline & $\mathrm{U}$ & 22 \\
\hline \multirow[t]{7}{*}{19841} & $\mathrm{U}$ & 93 \\
\hline & B & 39 \\
\hline & $\mathrm{F}$ & 31 \\
\hline & B & 19 \\
\hline & $\mathrm{F}$ & 15 \\
\hline & B & 15 \\
\hline & $\mathrm{U}$ & 15 \\
\hline \multirow[t]{6}{*}{14431} & U & 94 \\
\hline & B & 30 \\
\hline & $\mathrm{F}$ & 35 \\
\hline & B & 20 \\
\hline & $\mathrm{F}$ & 15 \\
\hline & U & 15 \\
\hline
\end{tabular}


Table 5

Experiment 3 Stimuli. Standard color refers to the key color corresponding with the respective multiple schedule component and Signal color refers to the key color during the during either the 1) DRO portion of the chain VT DRO schedule or 2) stimulus change in the Uncorrelated-Signal component.

\begin{tabular}{|c|c|c|c|c|}
\hline & \multicolumn{4}{|c|}{ Component } \\
\hline & \multicolumn{2}{|c|}{ Correlated Signal } & \multicolumn{2}{|c|}{ Uncorrelated Signal } \\
\hline Pigeon & Standard Color & Signal Color & Standard Color & Signal Color \\
\hline 14167 & Red & Green & Blue & White \\
\hline 14431 & Red & Green & Blue & White \\
\hline
\end{tabular}


Table 6

Experiment 3 Order of conditions and number of sessions for individual pigeons. $U=$ Unsignaled-Baseline condition, $F=$ Full-Signal condition.

\begin{tabular}{lll}
\hline Pigeon & Condition & Sessions \\
& & \\
\hline 14167 & U & 35 \\
& F & 44 \\
& U & 17 \\
\hline 14431 & U & 56 \\
& F & 39 \\
& U & 24 \\
\hline
\end{tabular}




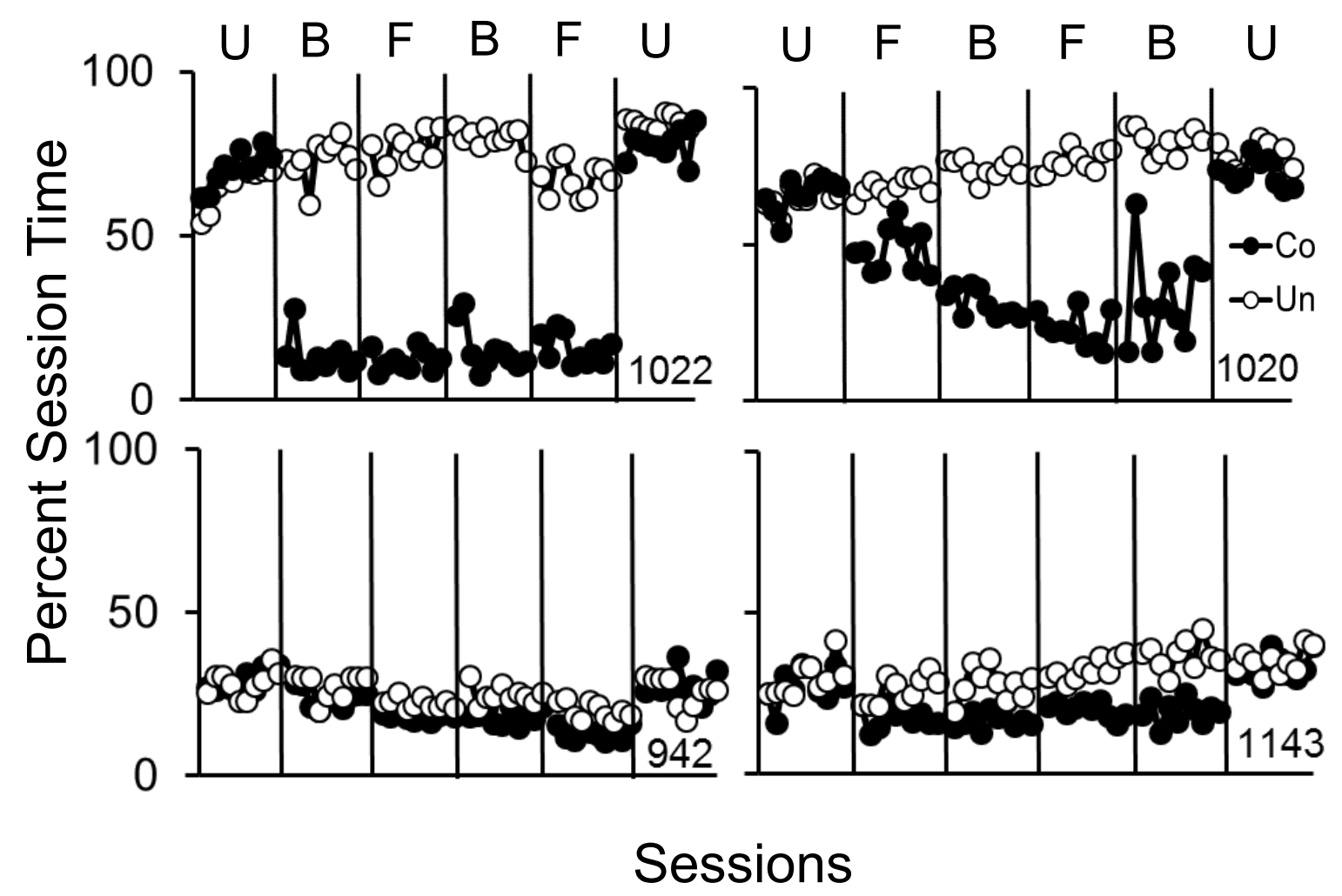

Figure 1. Percentage of session time in Experiment1 allocated to pausing in the UnsignaledBaseline (U), Brief-Signal (B), and Full-Signal conditions (F) for the last ten sessions, separated by Correlated-Signal (Co) and Uncorrelated-Signal (Un) components, for individual pigeons. 


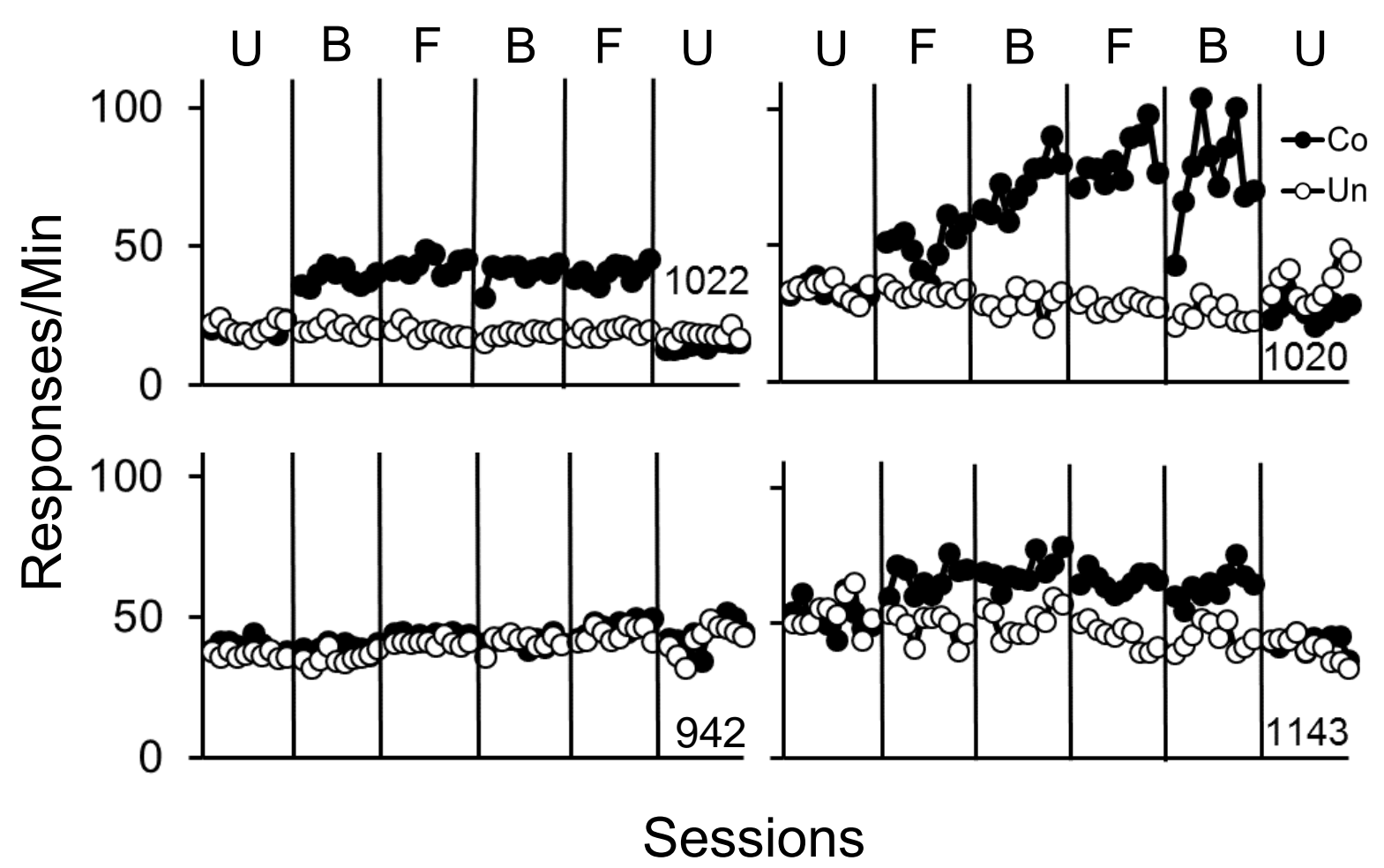

Figure 2. Response rates from Experiment 1 in the Unsignaled-Baseline (U), Brief-Signal (B), and Full-Signal conditions (F) for the last ten sessions, separated by Correlated-Signal (Co) and Uncorrelated-Signal (Un) components, for individual pigeons. 


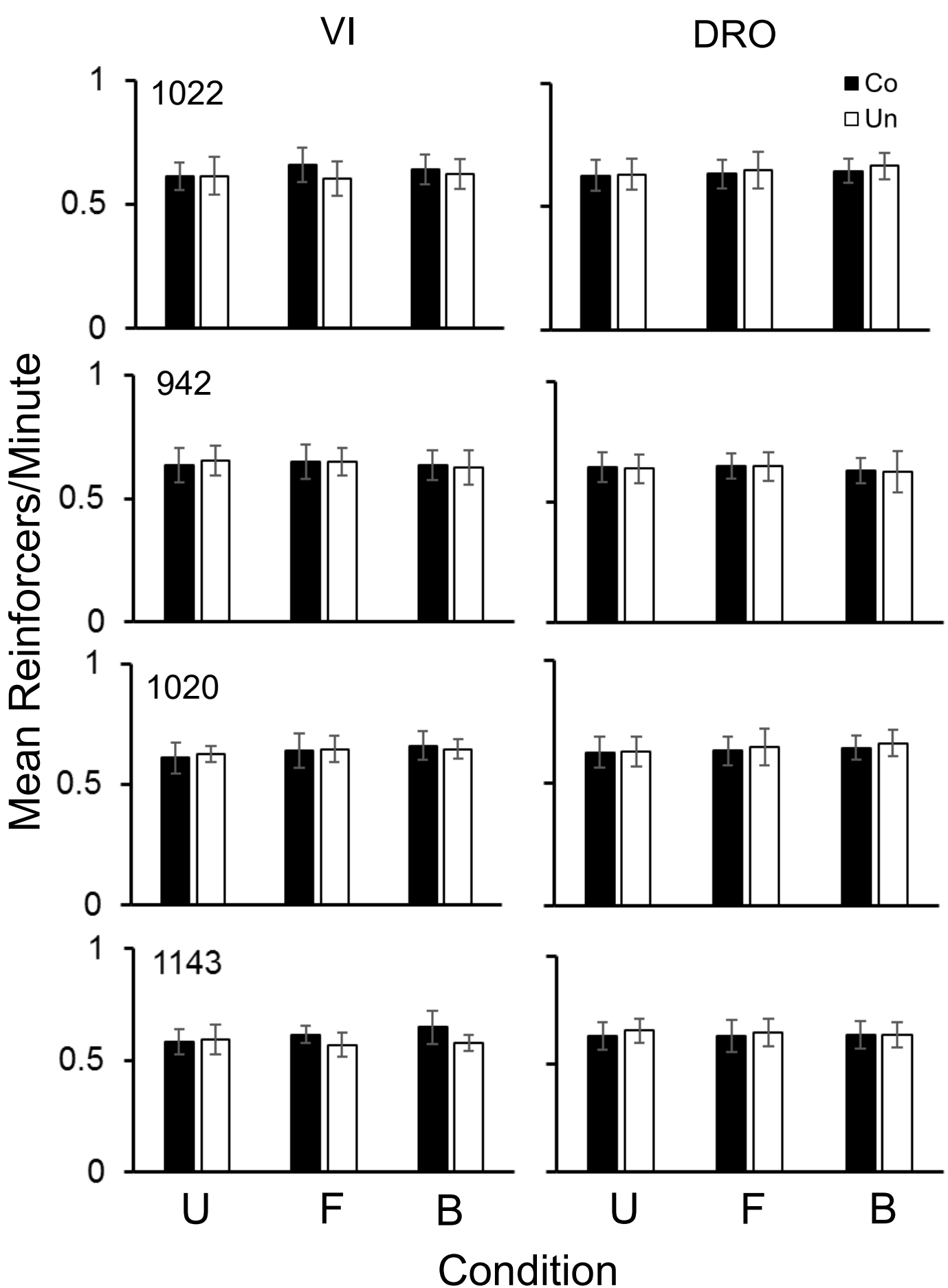

Figure 3. Mean reinforcement rates from Experiment 1 for the pecking (VI) and pausing (DRO) contingencies in the Unsignaled-Baseline (U), Brief-Signal (B), and Full-Signal conditions (F), separated by Correlated-Signal (Co) and Uncorrelated-Signal (Un) components. Error bars are the standard deviations. 


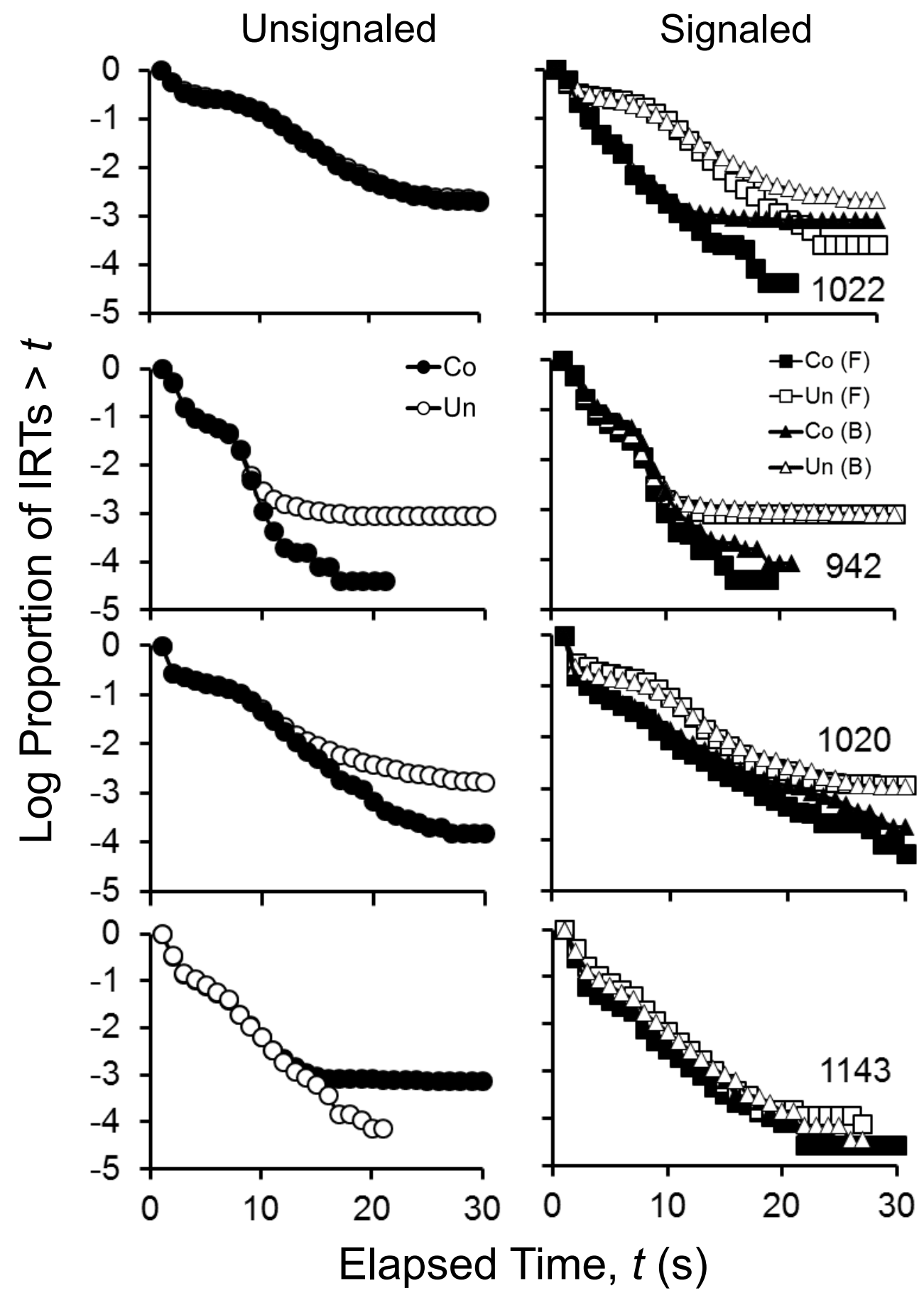

Figure 4. Log-survivor plots of IRTs from Experiment 1, separated by Correlated-Signal (Co) and Uncorrelated-Signal (Un) components during the Unsignaled-Baseline conditions, in the left panel, and from Full-Signal (F) and Brief-Signal (B) conditions, in the right panel. 


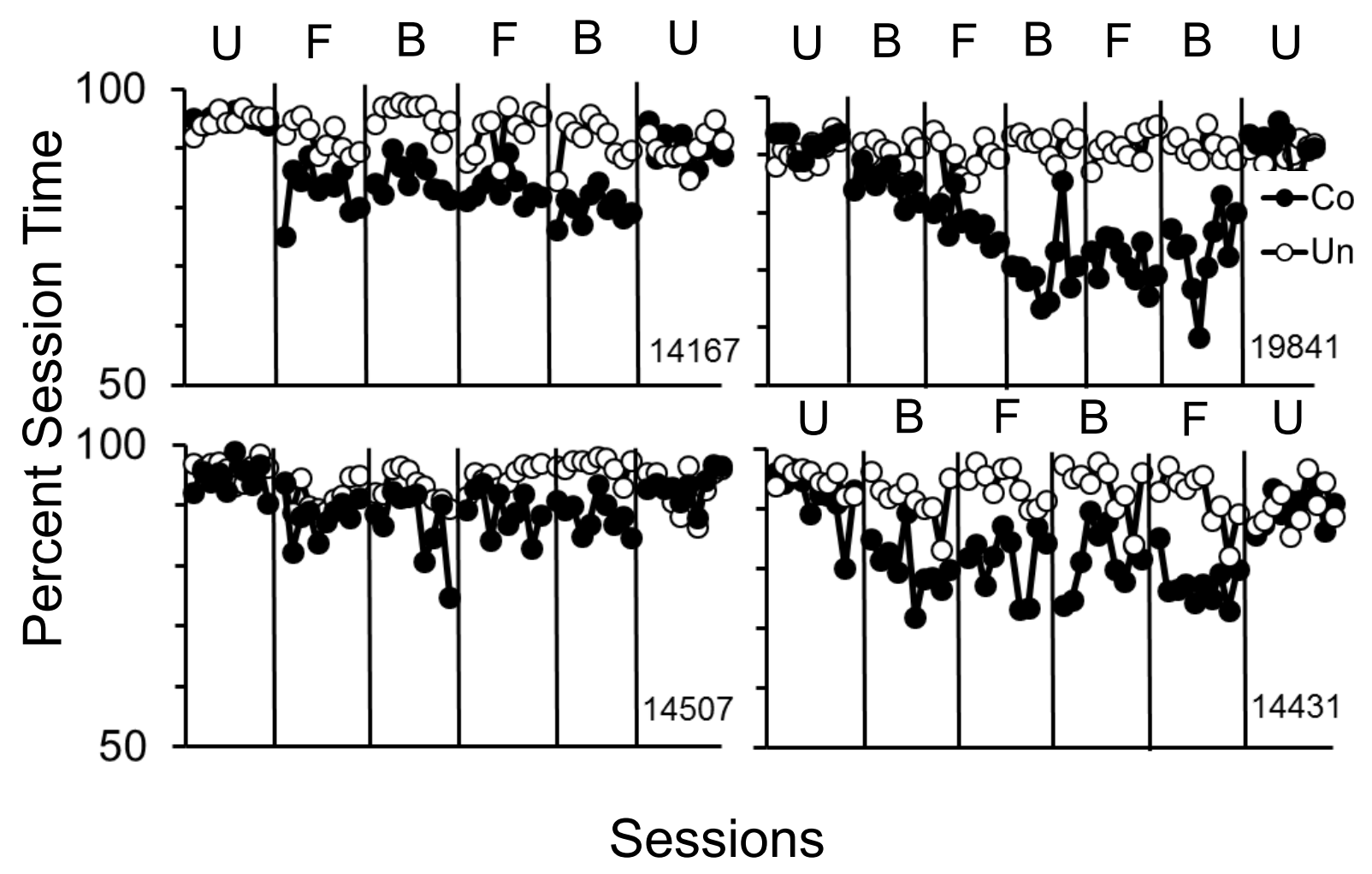

Figure 5. Percent time allocated to pausing from Experiment 2 in the Unsignaled-Baseline (U), Brief-Signal (B), and Full-Signal (F) conditions for the last ten sessions, separated by CorrelatedSignal (Co) and Uncorrelated-Signal (Un) components, for individual pigeons (y -axis starts at $50 \%)$. 


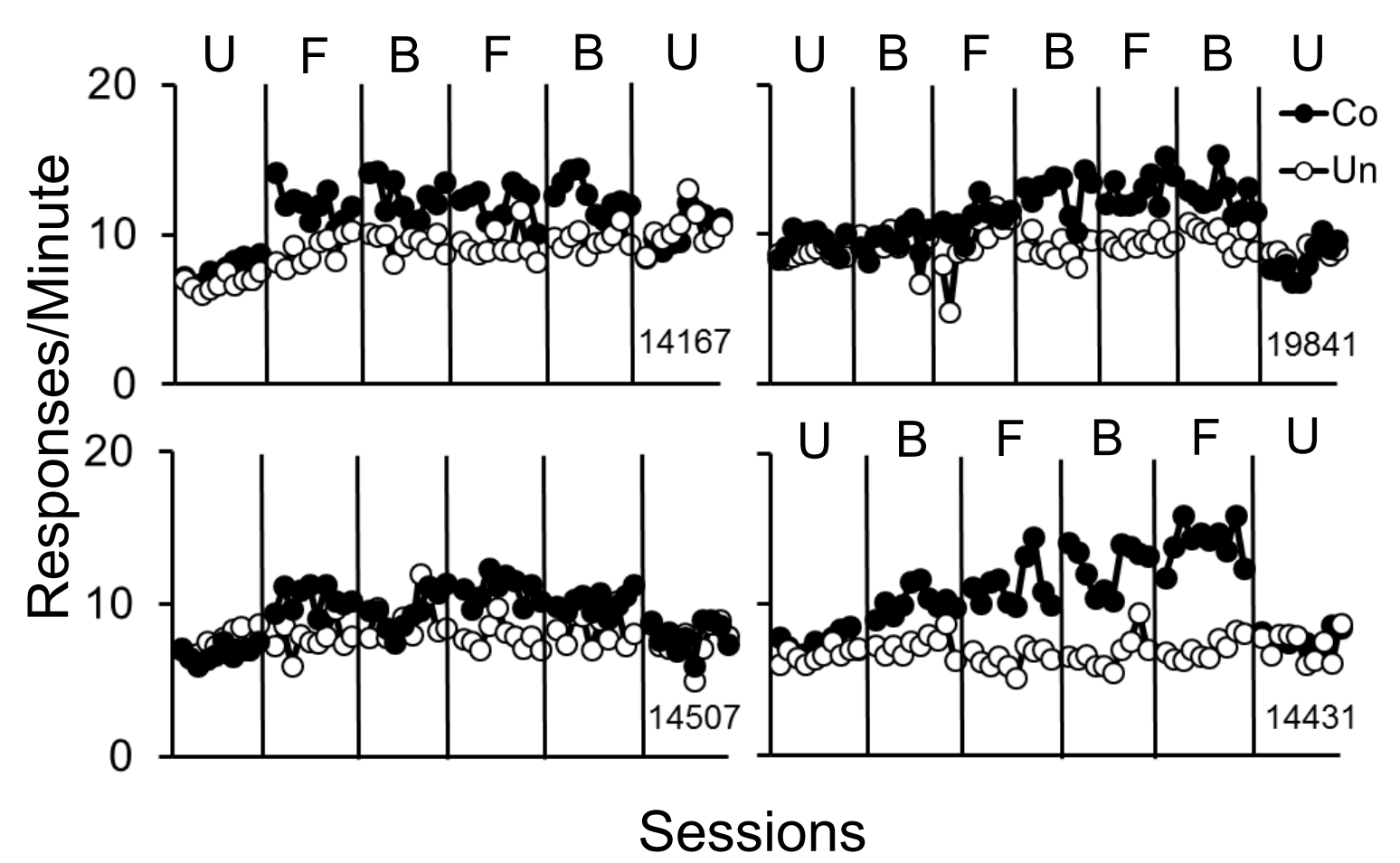

Figure 6. Response rates from Experiment 2 in the Unsignaled-Baseline (U), Brief-Signal (B), and Full-Signal conditions (F) for the last ten sessions, separated by Correlated-Signal (Co) and Uncorrelated-Signal (Un) components, for individual pigeons. 
DRL

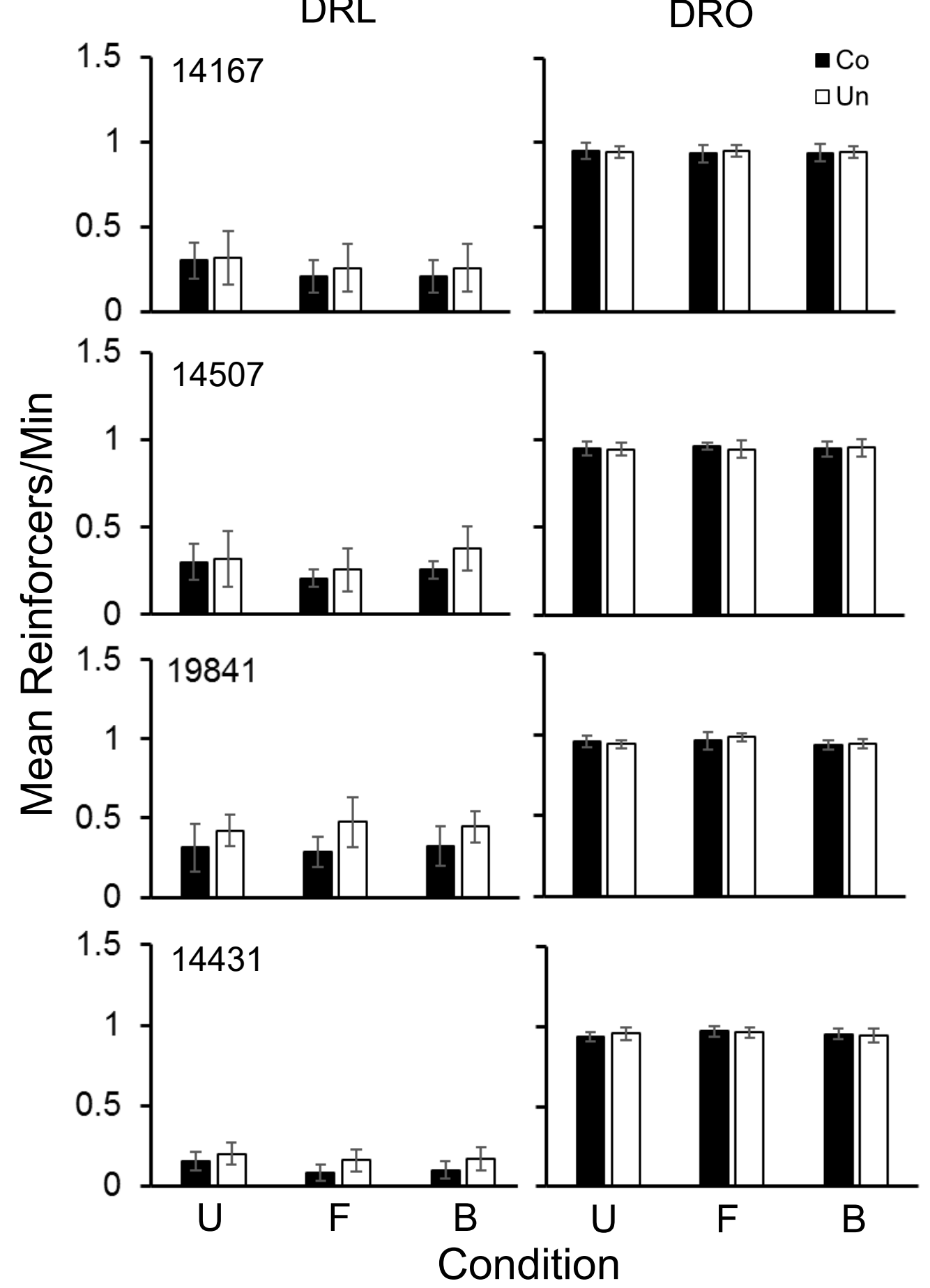

Figure 7. Mean reinforcement rates from Experiment 2 for the pecking (DRL) and pausing (DRO) contingencies in the Unsignaled-Baseline (U), Brief-Signal (B), and Full-Signal (F) conditions, separated by Correlated-Signal (Co) and Uncorrelated-Signal (Un) components. Error bars are the standard deviations. 


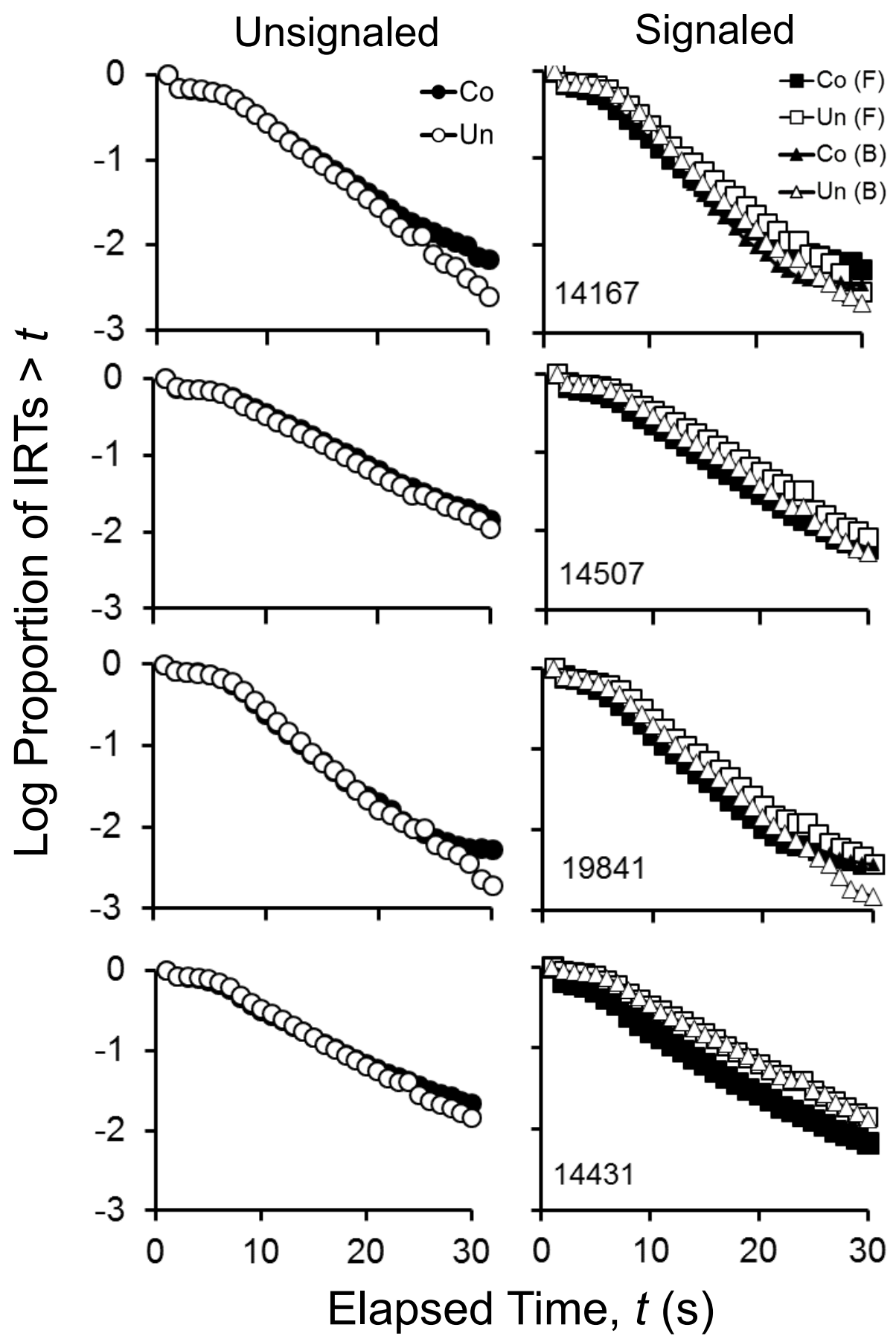

Figure 8. Log-survivor plots of IRTs from Experiment 2, separated by Correlated-Signal (Co) and Uncorrelated-Signal (Un) components during the Unsignaled-Baseline conditions, in the left panel, and from Full-Signal (F) and Brief-Signal (B) conditions, in the right panel. 


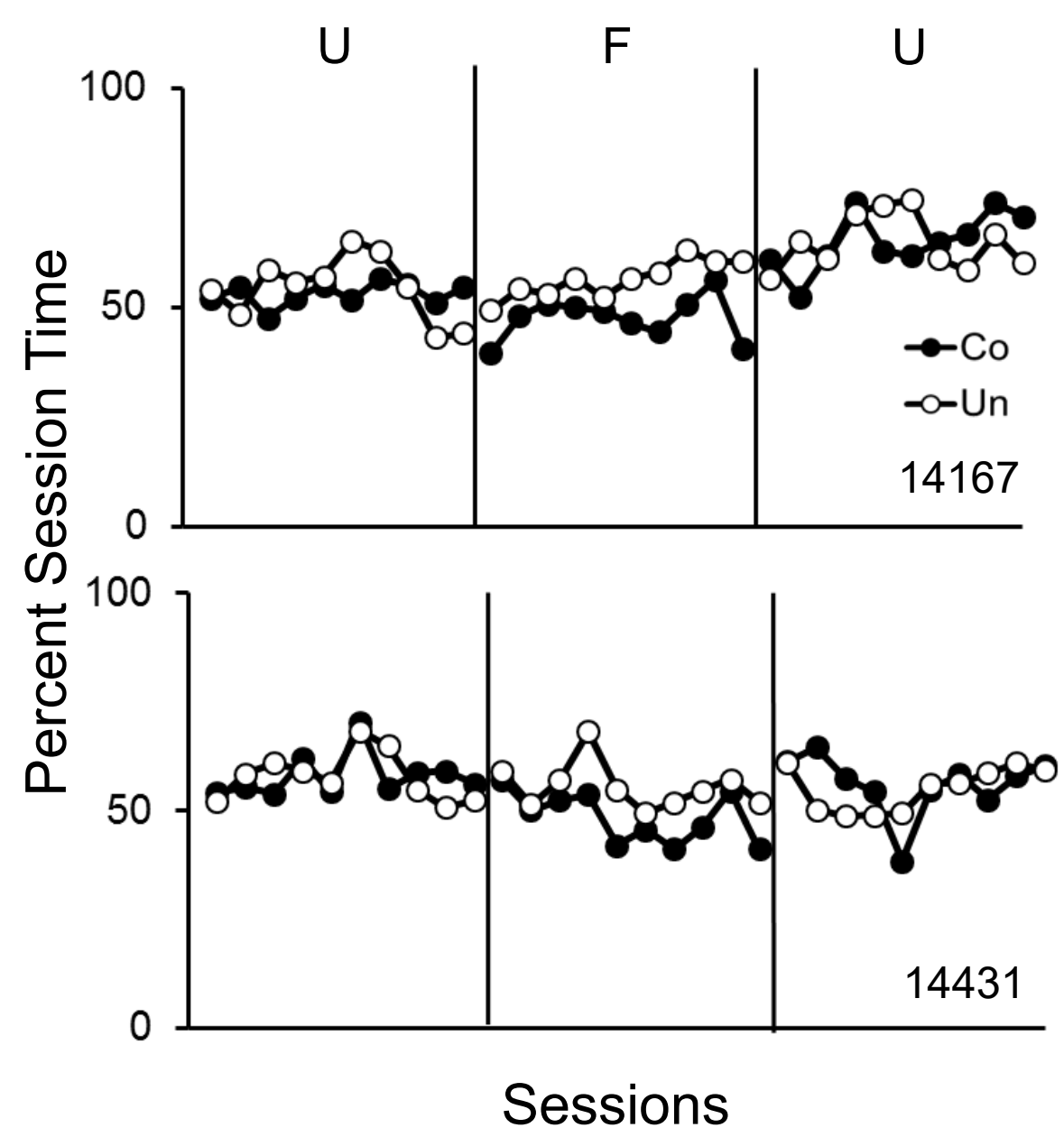

Figure 9. Percent session time allocated to pausing from Experiment 3 in the Unsignaled-

Baseline (U) and Full-Signal (F) conditions for the last ten sessions, separated by CorrelatedSignal (Co) and Uncorrelated-Signal (Un) components, for individual pigeons. 


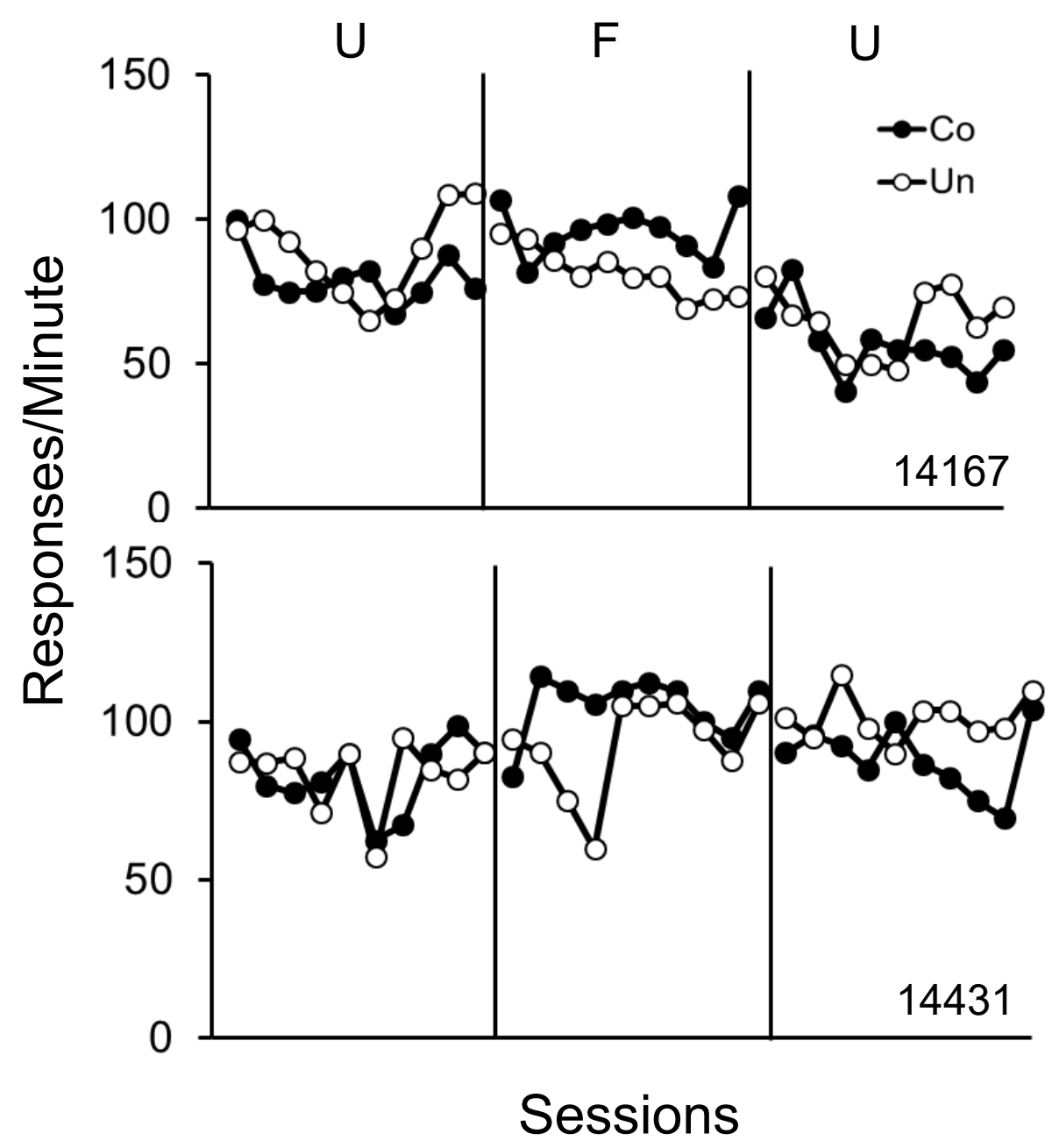

Figure 10. Response rates from Experiment 3 in the Unsignaled-Baseline (U) and Full-Signal (F) conditions for the last ten sessions, separated by Correlated-Signal (Co) and Uncorrelated-Signal (Un) components, for individual pigeons. 


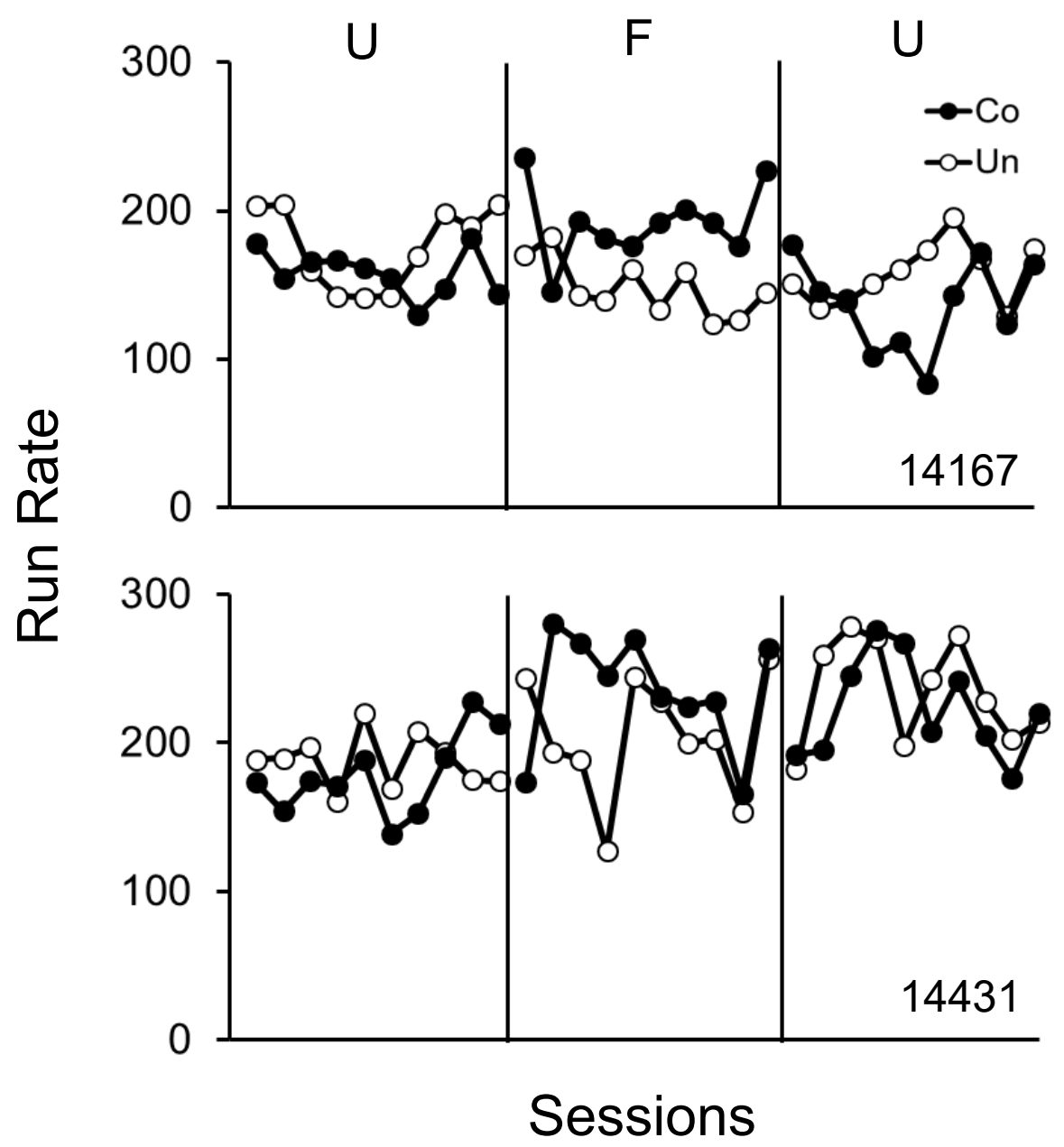

Figure 11. Run rates from Experiment 3 in the Unsignaled-Baseline (U) and Full-Signal (F) conditions, separated by Correlated-Signal (Co) and Uncorrelated-Signal (Un) components, for individual pigeons. 


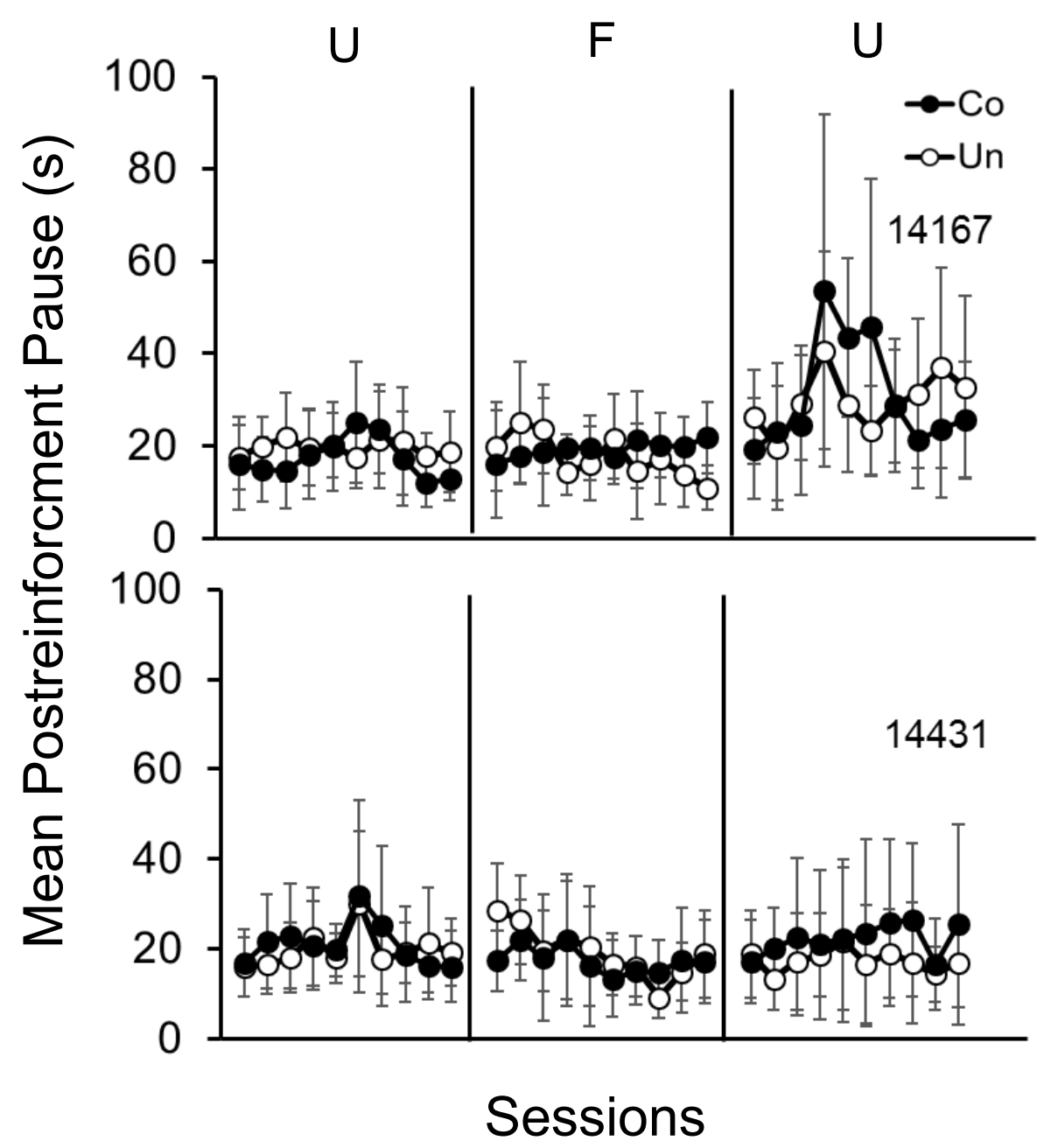

Figure 12. Mean postreinforcement pauses from Experiment 3 in the Unsignaled-Baseline (U) and Full-Signal (F) conditions, separated by Correlated-Signal (Co) and Uncorrelated-Signal (Un) components, for individual pigeons. Error bars are the standard deviations. 


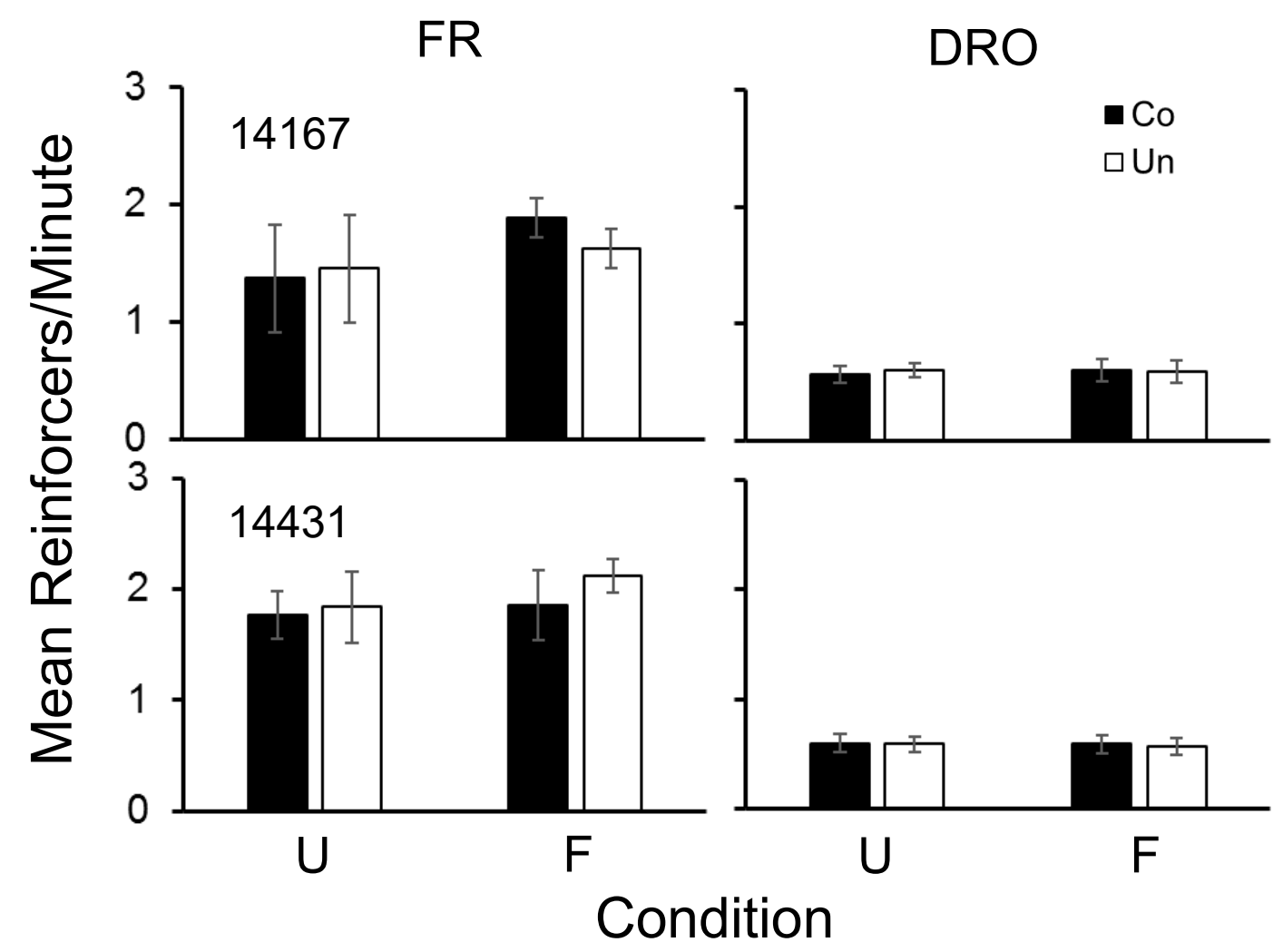

Figure 13. Mean reinforcement rates from Experiment 3 for the pecking (FR) and pausing (DRO) contingencies in the Unsignaled-Baseline (U) and Full-Signal (F) conditions, separated by Correlated-Signal (Co) and Uncorrelated-Signal (Un) components. Error bars are the standard deviations. 


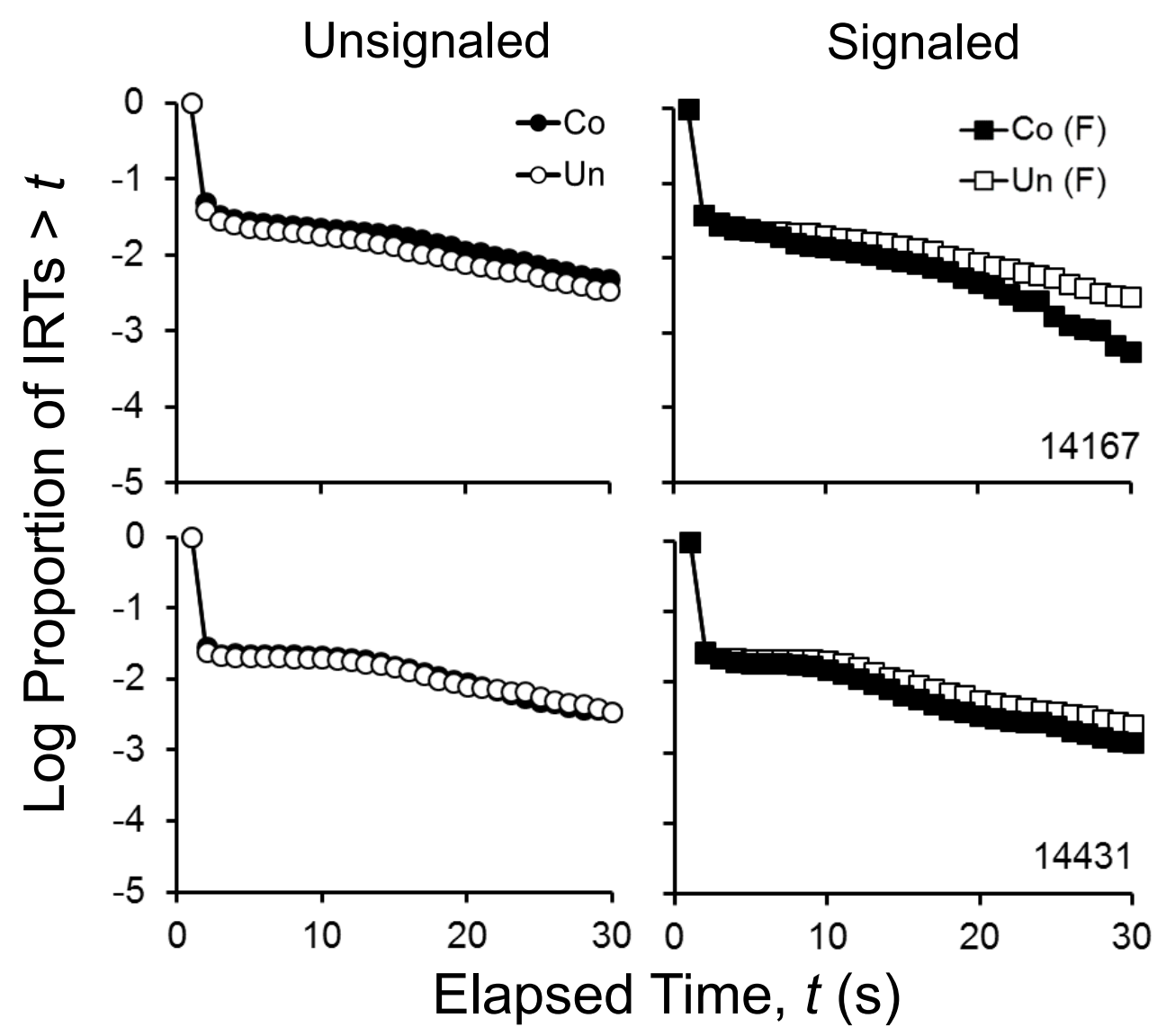

Figure 14. Log-survivor plots of IRTs from Experiment 3, separated by Correlated-Signal (Co) and Uncorrelated-Signal (Un) components during the Unsignaled-Baseline conditions, in the left panel, and from the Full-Signal (F) condition, in the right panel. 